\title{
PSEUDODIFFERENTIAL CALCULI ON THE HALF-LINE RESPECTING PRESCRIBED ASYMPTOTIC TYPES
}

\author{
Xiaochun Liu \\ School of Mathematics \\ and Statistics \\ Wuhan University \\ Wuhan, 430072 \\ P.R. China \\ liuxc72@public.wh.hb.cn
}

\author{
Ingo Witt \\ Institute of Mathematics \\ University of Potsdam \\ D-14415 Potsdam \\ Germany \\ ingo@math.uni-potsdam.de
}

1. INTRODUCTION

A function $u(t)$ on $\mathbb{R}_{+}$is said to possess conormal asymptotics if

$$
u(t) \sim \sum_{j=1}^{M} \sum_{k=0}^{m_{j}} t^{-p_{j}} \log ^{k} t c_{j k} \text { as } t \rightarrow+0,
$$

where $M \in \mathbb{N} \cup\{\infty\}, p_{j} \in \mathbb{C}, \operatorname{Re} p_{j} \rightarrow-\infty$ as $j \rightarrow \infty$ when $M=\infty, m_{j} \in \mathbb{N}$, and $c_{j k} \in \mathbb{C}$. Here, the asymptotics are understood in an increasing order 
of flatness at $t=0$. An asymptotic type, $P$, is represented by a sequence $\left\{\left(p_{j}, m_{j}\right)\right\}_{j=1}^{M}$ grouping together the singular exponents, $p_{j}$, and their multiplicities, $m_{j}$. In fact, an asymptotic type is regarded as a non-negative divisor on $\mathbb{C}$ possessing special support properties (see Definitions $2.1,2.3$ ). If the data in (1.1) are coming from $P$, then $u(t)$ is said to possess conormal asymptotics of type $P$.

The goal of this paper is to construct classes $\Psi_{P, Q}^{\mu, d}\left(\mathbb{R}_{+}\right) \subset L_{\mathrm{cl}}^{\mu}\left(\mathbb{R}_{+}\right) \cap L^{\mu ; 0}\left(\mathbb{R}_{+}\right)$for $\mu \in \mathbb{Z}$ (where superscript " 0 " in the second space refers to the exit behaviour as $t \rightarrow \infty)$ of pseudodifferential operators $A$ on the half-line $\mathbb{R}_{+}$such that, if $u(t)$ possess conormal asymptotics of type $P$, then $A u(t)$ possess conormal asymptotics of type $Q$. Here, $\mu \in \mathbb{Z}$ is the order of operators involved, $P, Q$ are prescribed asymptotic types, and $d \in \mathbb{N}$ is a type for these operators similar to that in Boutet de Monvel's calculus for pseudodifferential boundary value problems arising, e.g., in compositions, see Boutet DE Monvel [1], Rempel-Schulze [18], Schulze [23]. Boutet de Monvel's calculus, indeed, appears as a special case when $P=Q$ is the asymptotic type resulting from Taylor expansion at $t=0$.

Throughout, we shall fix a conormal order $\delta \in \mathbb{R}$ (in an $L^{2}$-sense) as basic reference weight. In (1.1), we then have $\operatorname{Re} p_{j}<1 / 2-\delta$ for all $j$.

In the primary case, $d=0$, we construct $\Psi_{P, Q}^{\mu, 0}\left(\mathbb{R}_{+}\right)$as a certain subclass of Schulze's cone calculus $C^{\mu}\left(\mathbb{R}_{+} ; \delta, \delta-\mu^{+}\right)$, for the latter, see Schulze [22, 23] and Appendix A. Since now, compared to the situation occurring for general cone pseudodifferential operators in $C^{\mu}\left(\mathbb{R}_{+} ; \delta, \delta-\mu^{+}\right)$, asymptotic types are fixed, we may allow for conditions of trace and potential type at $t=0$; thus, leading to classes $\Psi_{P, Q}^{\mu, d}\left(\mathbb{R}_{+} ; N_{-}, N_{+}\right)$with $N_{-}$being the number of trace conditions and $N_{+}$being the number of potential conditions, respectively. In particular, $\Psi_{P, Q}^{\mu, d}\left(\mathbb{R}_{+}\right)=\Psi_{P, Q}^{\mu, d}\left(\mathbb{R}_{+} ; 0,0\right)$.

Apart from the construction of the classes $\Psi_{P, Q}^{\mu, d}\left(\mathbb{R}_{+} ; N_{-}, N_{+}\right)$, let us now state the main results of this paper: For $s \geq 0$, let $\mathbb{H}_{P}^{s, \delta}\left(\mathbb{R}_{+}\right) \subset H_{\mathrm{loc}}^{s}\left(\mathbb{R}_{+}\right)$be the 
Hilbert space, as introduced in Section 2.3 below, of functions on $\mathbb{R}_{+}$possessing conormal asymptotics of type $P$ as $t \rightarrow+0$, where asymptotics are observed on a weight interval of length $s$. (For details, see there.)

Theorem 1.1. There is non-decreasing sequence $\left\{s_{d}\right\}_{d \geq d_{0}} \subset \mathbb{R}_{+}$depending on $P($ see $(3.6))$, where $d_{0} \geq 1$, such that each $\mathcal{A} \in \Psi_{P, Q}^{\mu, d}\left(\mathbb{R}_{+} ; N_{-}, N_{+}\right)$induces a continuous operator

$$
\begin{aligned}
& \mathbb{H}_{P}^{s, \delta}\left(\mathbb{R}_{+}\right) \quad \mathbb{H}_{Q}^{s-\mu, \delta}\left(\mathbb{R}_{+}\right) \\
& \mathcal{A}: \quad \oplus \quad \rightarrow \quad \oplus \\
& \mathbb{C}^{N_{-}} \quad \mathbb{C}^{N_{+}}
\end{aligned}
$$

for all $s$ satisfying $s \geq \mu^{+}$and, in addition, $s>s_{d}$ when $d \geq d_{0}$.

Theorem 1.2. For $\mathcal{A} \in \Psi_{\tilde{Q}, Q}^{\mu, d}\left(\mathbb{R}_{+} ; \tilde{N}, N_{+}\right), \mathcal{B} \in \Psi_{P, \tilde{Q}}^{\mu^{\prime}, d^{\prime}}\left(\mathbb{R}_{+} ; N_{-}, \tilde{N}\right)$, composition yields

$$
\mathcal{A B} \in \Psi_{P, Q}^{\mu+\mu^{\prime}, d^{\prime \prime}}\left(\mathbb{R}_{+} ; N_{-}, N_{+}\right),
$$

where $d^{\prime \prime}=\max \left\{d+\mu^{\prime}, d^{\prime}\right\}$.

Let $\Sigma_{P, Q}^{\mu}:=\left\{\left(\sigma_{M}^{\mu}(\mathcal{A}), \sigma_{\psi}^{\mu}(\mathcal{A})\right) ; \mathcal{A} \in \Psi_{P, Q}^{\mu, d}\left(\mathbb{R}_{+} ; N_{-}, N_{+}\right)\right\}$be the principal symbol space for the operator space $\Psi_{P, Q}^{\mu, d}\left(\mathbb{R}_{+} ; N_{-}, N_{+}\right)$. (An intrinsic definition is provided in (4.1).) Here, $\sigma_{M}^{\mu}(\mathcal{A})(z)$, where $z \in \mathbb{C}$, is the Mellin principal symbol and $\sigma_{\psi}^{\mu}(\mathcal{A})(t, \tau)$, where $(t, \tau) \in \overline{\mathbb{R}}_{+} \times(\mathbb{R} \backslash 0)$, is the interior principal symbol for an operator $\mathcal{A} \in \Psi_{P, Q}^{\mu, d}\left(\mathbb{R}_{+} ; N_{-}, N_{+}\right)$. (Since we are interested in the behaviour as $t \rightarrow+0$, the principal exit symbol $\sigma_{e}^{0}(\mathcal{A})$ that controls the behaviour as $t \rightarrow \infty$ is excluded from the considerations.)

Theorem 1.3. The short sequence

$$
0 \longrightarrow \Psi_{P, Q}^{\mu-1, d}\left(\mathbb{R}_{+} ; N_{-}, N_{+}\right) \longrightarrow \Psi_{P, Q}^{\mu, d}\left(\mathbb{R}_{+} ; N_{-}, N_{+}\right) \stackrel{\left(\sigma_{M}^{\mu}, \sigma_{\psi}^{\mu}\right)}{\longrightarrow} \Sigma_{P, Q}^{\mu} \longrightarrow 0
$$
is split exact. 
An operator $\mathcal{A} \in \Psi_{P, Q}^{\mu, d}\left(\mathbb{R}_{+} ; N_{-}, N_{+}\right)$is called elliptic if the principal divisor of its Mellin principal divisor $\sigma_{M}^{\mu}(\mathcal{A})(z)$ equals $P-T^{-\mu} Q$, where the asymptotic types $P, Q$ are regarded as divisors and $T^{-\mu}$ is the shift by $\mu$ to the left (see Definition 4.2). Otherwise, the usual ellipticity conditions for $\sigma_{\psi}^{\mu}(\mathcal{A}), \sigma_{e}^{0}(\mathcal{A})$ are assumed. Note that the first of these ellipticity conditions is stronger than that in Schulze's cone calculus (see Definition A.6(i)) in as far that, now, not only a condition along one vertical (weight) line in $\mathbb{C}$ is imposed, but a condition on the whole of $\mathbb{C}$. (This point is further discussed in Remarks 3.17, 4.3(a), and 4.11.)

Theorem 1.4. Let $\mathcal{A} \in \Psi_{P, Q}^{\mu, d}\left(\mathbb{R}_{+} ; N_{-}, N_{+}\right)$be elliptic. Then $A$ regarded as operator in (1.2) is a Fredholm operator for all $s$ satisfying $s \geq \mu^{+}$, and $s>s_{d}$ when $d \geq d_{0}$. Furthermore, in this case, there is a parametrix $\mathcal{P}$ to $\mathcal{A}$, i.e., an operator $\mathcal{P} \in \Psi_{Q, P}^{-\mu, 0}\left(\mathbb{R}_{+} ; N_{+}, N_{-}\right)$such that

$$
\mathcal{P} \mathcal{A}-1 \in \Psi_{G ; P}^{\max \{\mu, d\}}\left(\mathbb{R}_{+} ; N_{-}, N_{-}\right), \quad \mathcal{A} \mathcal{P}-1 \in \Psi_{G ; Q}^{(d-\mu)^{+}}\left(\mathbb{R}_{+} ; N_{+}, N_{+}\right) .
$$

Here, $\Psi_{G ; P, Q}^{d}\left(\mathbb{R}_{+} ; N_{-}, N_{+}\right)$denotes the class of Green operators of type $d$, i.e., the class of residual elements within the calculus (see Definition 3.10). In particular, $\Psi_{G ; P, Q}^{d}\left(\mathbb{R}_{+}\right) \subset L^{-\infty ;-\infty}\left(\mathbb{R}_{+}\right)$. We also agree to write $\Psi_{G ; P}^{d}\left(\mathbb{R}_{+} ; N_{-}, N_{+}\right)$ instead of $\Psi_{G: P, P}^{d}\left(\mathbb{R}_{+} ; N_{-}, N_{+}\right)$.

Theorem 1.5. Let $\mathcal{A} \in \Psi_{P, Q}^{\mu, d}\left(\mathbb{R}_{+} ; N_{-}, N_{+}\right)$be elliptic. Assume that $\mathcal{A}$ regarded as operator in (1.2) is invertible for some $s$ satisfying $s \geq \mu^{+}$, and $s>s_{d}$ when $d \geq d_{0}$. Then $\mathcal{A}$ regarded as operator in (1.2) is invertible for all $s$ satisfying $s \geq \mu^{+}$, and $s>s_{d}$ when $d \geq d_{0}$, and its inverse $\mathcal{A}^{-1}$ belongs to $\Psi_{Q, P}^{-\mu,(d-\mu)^{+}}\left(\mathbb{R}_{+} ; N_{+}, N_{-}\right)$.

Finally, we come to the coordinate invariance of the calculus: Let $\chi: \overline{\mathbb{R}}_{+} \rightarrow \overline{\mathbb{R}}_{+}$ be a $C^{\infty}$-diffeomorphism such that $\left|\partial_{t}^{j} \chi(t)\right| \leq C_{j}(1+t)^{1-j}$ for all $t \in \overline{\mathbb{R}}_{+}, j \in \mathbb{N}$, and some constants $C_{j}>0$ and $|\chi(t)| \geq c t$ for all $t \in \overline{\mathbb{R}}_{+}$and some constant $c>0$. 
Theorem 1.6. Assume that $P, Q \in \underline{A s}^{\delta}$ satisfy the shadow condition (see Definition 2.3). Then, for each $\mathcal{A} \in \Psi_{P, Q}^{\mu, d}\left(\mathbb{R}_{+} ; N_{-}, N_{+}\right)$, the operator pushforward $\mathcal{A}_{\chi}$ of $\mathcal{A}$ under the diffeomorphism $\chi$ belongs to $\Psi_{P, Q}^{\mu, d}\left(\mathbb{R}_{+} ; N_{-}, N_{+}\right)$.

There is a number of related work to mention: The constructions rely on Schulze's cone calculi on the half-line $\mathbb{R}_{+}$, see ScHulzE $[21,22,23]$, and are oriented towards Boutet de Monvel's calculus for pseudodifferential boundary problems, see BouteT DE MONVEL [1]. The basic idea of choosing the complete Mellin symbol in an appropriate way is from WITT [26], the essential result allowing to construct corresponding Mellin symbols is from WiTT [27]. A first version of the calculus ( $P=Q$, no coordinate invariance) has been worked out by LIU [13] in her thesis. To incorporate coordinate invariance into the calculus has become possible after explicit formulas for coordinate changes for Mellin symbols have been established in KAPANADZE-SCHulzEWiTT [10].

An extension of these results to higher-dimensional cones probably requires a finer observation of asymptotic types (including linear relations among the various coefficients $c_{j k}$ in (1.1) which are then smooth functions on the cone basis). The abstract concept for establishing corresponding asymptotic types, that extends concepts from REMPEL-Schulze [19] and Schulze [21], is found in WITT [29]. The concrete computations for higher-dimensional cones have been performed in LiU-WiTT [14].

The paper is organized as follows: In Section 2, some auxiliary material (e.g., asymptotic types, Mellin symbols, function spaces with asymptotics) is presented. In Section 3, the calculus is introduced and its basic elements (e.g., continuity in the above-mentioned function spaces, compositions) are developed. Then, in Section 4, further elements of the calculus (e.g., principal symbol map, ellipticity, parametrix construction, coordinate invariance) are 
deal with. Finally, in Appendix A, Schulze's cone calculus on the half-line $\mathbb{R}_{+}$ that is basic for all following constructions is reviewed.

Notation. We shall employ standard notation from microlocal analysis as found, e.g., in Hörmander [8], Kumano-Go [12], and Schulze [23].

Furthermore, we shall use the following notation: $\Gamma_{\beta}:=\{z \in \mathbb{C} ; \operatorname{Re} z=\beta\}$ for $\beta \in \mathbb{R}$ denotes a vertical line in the complex plane. For $\alpha, \beta \in \mathbb{R}, \alpha<\beta$, we set $S(\alpha, \beta):=\{z \in \mathbb{C} ; \alpha<\operatorname{Re} z<\beta\}$ and $S(\beta):=\{z \in \mathbb{C} ; \operatorname{Re} z<\beta\} . \mathcal{A}(S(\alpha, \beta))$ is the space of all holomorphic functions on the strip $S(\alpha, \beta)$. The Mellin transformation $M u(z)=\tilde{u}(z):=\int_{0}^{\infty} t^{z-1} u(t) d t$, first defined for $u \in C_{\mathrm{comp}}^{\infty}\left(\mathbb{R}_{+}\right)$, extends to other function spaces by continuity. Its inverse transformation is given by $M^{-1} v(t)=\frac{1}{2 \pi i} \int_{\Gamma_{\beta}} t^{-z} v(z) d z$, with $\beta \in \mathbb{R}$ appropriately chosen. For $h=h(t, z)$ being a function on $\mathbb{R}_{+} \times \Gamma_{\beta}$ for some $\beta \in \mathbb{R}$, op ${ }_{M}^{\beta}(h)$ means the pseudodifferential operator

$$
\begin{aligned}
\operatorname{op}_{M}^{\beta}(h) u(t):=M_{z \rightarrow t}^{-1}\{h(t, z) \tilde{u}(z)\} \\
=\frac{1}{2 \pi i} \int_{\Gamma_{\beta}} \int_{0}^{\infty}\left(\frac{t}{t^{\prime}}\right)^{-z} h(t, z) u\left(t^{\prime}\right) \frac{d t^{\prime}}{t^{\prime}}, \quad t>0 .
\end{aligned}
$$

Similarly, op $\psi(p) u(t):=F^{-1}\{p(t, \tau) \hat{u}(\tau)\}=\frac{1}{2 \pi} \iint_{\mathbb{R} \times \mathbb{R}} e^{i\left(t-t^{\prime}\right) \tau} p(t, \tau) u\left(t^{\prime}\right) d t^{\prime} d \tau$, $t \in \mathbb{R}$, for functions $p=p(t, \tau)$ defined on $\mathbb{R} \times \mathbb{R}$, where $F u(\tau)=\hat{u}(\tau):=$ $\int_{\mathbb{R}} e^{-i t \tau} u(t) d t$ is the Fourier transformation. $S^{\mu ; \nu}\left(\mathbb{R}_{+} \times \mathbb{R}\right)$ for $\mu, \nu \in \mathbb{Z}$ denotes the class of all symbols $p=p(t, \tau)$ of order $\mu$ and type 1,0 on $\mathbb{R}_{+} \times \mathbb{R}$ satisfying an exit condition of order $\nu$ as $t \rightarrow \infty$, i.e., for all $j, k \in \mathbb{N}, \delta>0$, there is a constant $C_{j k \delta}>0$ such that

$$
\left|\partial_{t}^{k} \partial_{\tau}^{j} p(t, \tau)\right| \leq C_{j k \delta}(1+|\tau|)^{\mu-j}(1+t)^{\nu-k}, \quad(t, \tau) \in \mathbb{R}_{+} \times \mathbb{R}, t \geq \delta,
$$

and $S^{-\infty ;-\infty}\left(\mathbb{R}_{+} \times \mathbb{R}\right):=\bigcap_{\mu, \nu} S^{\mu ; \nu}\left(\mathbb{R}_{+} \times \mathbb{R}\right)$. Then, $S^{(\mu) ; 0}\left(\overline{\mathbb{R}}_{+} \times(\mathbb{R} \backslash 0)\right)$ is the corresponding class of symbols which are positively homogeneous of degree $\mu$ in $\tau$ and smooth up to $t=0$. The class of pseudodifferential operators on $\mathbb{R}_{+}$corresponding to $S^{\mu ; 0}\left(\mathbb{R}_{+} \times \mathbb{R}\right)$ is denoted by $L^{\mu ; 0}\left(\mathbb{R}_{+}\right)$. op op $_{G}(g)$ for 
$g \in C^{\infty}\left(\mathbb{R}_{+} \times \mathbb{R}_{+}\right)$is the operator given by op ${ }_{G}(g) u(t)=\int_{0}^{\infty} g\left(t, t^{\prime}\right) u\left(t^{\prime}\right) t^{\prime-2 \delta} d t^{\prime}$, $u \in C_{\text {comp }}^{\infty}\left(\mathbb{R}_{+}\right)$, where $\delta \in \mathbb{R}$ is the fixed conormal order (see (2.8)). $\omega, \omega_{0}, \omega_{1}$ will always be cut-off functions on $\mathbb{R}_{+}$, i.e., $\omega, \omega_{0}, \omega_{1} \in C^{\infty}\left(\overline{\mathbb{R}}_{+}\right)$have bounded support and $\omega(t)=1$ for $t$ close to 0 , similarly for $\omega_{0}, \omega_{1}$, satisfying $\omega \omega_{0}=\omega$, $\omega \omega_{1}=\omega_{1}$.

\section{Preliminaries}

In this section, basic notions and some auxiliary material are introduced.

2.1. Asymptotic types. Asymptotic types allow to integrate formal asymptotic expansions into a functional-analytic setting. This idea goes back to ReMPEL-Schulze [19].

Definition 2.1. An asymptotic type, $P$, is a non-negative divisor on $\mathbb{C}$ satisfying $|\operatorname{Re} p| \rightarrow \infty$ as $|p| \rightarrow \infty$ on $\operatorname{supp} P:=\{p \in \mathbb{C} ; P(p) \neq 0\}$. The set of all asymptotic types is denoted by $\underline{\text { As. }}$.

The set $\underline{A s}$ is an ordered semigroup that is a lattice. It obeys the additional property that each non-empty subset (each bounded subset) has a greatest lower bound (a least upper bound). The order relation on the divisor group restricted to $\underline{\text { As }}$ is denoted by “ß”.

The least element of $\underline{A s}$ is the zero divisor, $\mathcal{O}$, and it is called the empty asymptotic type.

We shall employ the shift operation on asymptotic types that is defined by $\left(T^{\rho} P\right)(p)=P(p+\rho)$ for $P \in \underline{\text { As }}, \rho \in \mathbb{R}$.

If convenient, an asymptotic type, $P$, will be also represented by a sequence $\left\{\left(p_{j}, m_{j}\right)\right\}_{j=1}^{M} \subset \mathbb{C} \times \mathbb{N}$, where $M \in \mathbb{N} \cup\{\infty\}$ and $\left|\operatorname{Re} p_{j}\right| \rightarrow \infty$ as $j \rightarrow \infty$ when $M=\infty$, such that

$$
P(p)=\max \left\{m_{j}\right\}+1, \quad p \in \operatorname{supp} P,
$$


and the maximum is extended over all $j$ satisfying $p_{j}=p$. (Notice that taking plus 1 is convention, see, e.g., SchulzE [21, 23].) Of course, such a representation is not unique, although, by virtue of (2.1), each sequence represents a uniquely determined asymptotic type, $P$.

For conormal asymptotics as $t \rightarrow+0$ of functions $u=u(t)$ on $\mathbb{R}_{+}$only a restricted class of asymptotic types occurs. (Though, the generality introduced above is needed for Mellin symbols.)

Example 2.2. $P_{0}:=\{(-j, 0)\}_{j \in \mathbb{N}}$ is the asymptotic type resulting from Taylor expansion at $t=0$. (See (2.7). Note the minus sign in front of the $j$ that is related to the convention adopted for the Mellin transformation.)

Throughout, we shall fix a conormal order $\delta \in \mathbb{R}$ as basic reference weight. By $\underline{A s}^{\delta}$ we denote the set of all $P \in \underline{A s}$ such that $\operatorname{supp} P \subset S(1 / 2-\delta)$.

Definition 2.3. Given $\delta \in \mathbb{R}$, the set $\underline{A s^{\sharp}}$ consists of all $P \in \underline{A s}{ }^{\delta}$ such that $\operatorname{supp} P \cap \Gamma_{1 / 2-\delta-d}=\emptyset$ for all $d \in \mathbb{N}$ and the shadow condition $T^{-1} P \preccurlyeq P$ is satisfied.

In case it is necessary to mention the dependence on $\delta$ explicitly, we write $\underline{A s}{ }^{\sharp ; \delta}$.

For any meromorphic function $h$ on $\mathbb{C},(h(z))$ denotes the principal divisor assigned to it. In particular, $(h(z)) \succcurlyeq 0$ if and only if $h$ is holomorphic on $\mathbb{C}$ (i.e., it is an entire function). $(h(z))=P-Q$ for asymptotic types $P, Q$ means that $h$ has zeros given by $P$ and poles given by $Q$ with possible cancellation between both.

2.2. Mellin symbols. In the following, the complex Mellin variable $z \in \mathbb{C}$ is split into $z=\beta+i \tau$, where $\beta, \tau \in \mathbb{R}$.

Definition 2.4. For $P=\left\{\left(p_{j}, m_{j}\right)\right\}_{j=1}^{M} \in \underline{\text { As}}, \mu \in \mathbb{R} \cup\{-\infty\}, \mathcal{M}_{P}^{\mu}$ denotes the space of all meromorphic functions $h(z)$ on $\mathbb{C}$ having poles at most at 
$z=p_{j}$ of order $m_{j}$, for any $j$, such that, for all $\chi \in C^{\infty}(\mathbb{C})$ with $\chi(z)=1$ if $\operatorname{dist}(z, \operatorname{supp} P) \geq 1, \chi(z)=0$ if $\operatorname{dist}(z, \operatorname{supp} P) \leq 1 / 2$, we have $\left.(\chi h)(z)\right|_{z=\beta+i \tau} \in$ $C^{\infty}\left(\mathbb{R}_{\beta}, S_{\text {cl }}^{\mu}\left(\mathbb{R}_{\tau}\right)\right)$.

We also introduce

$$
\mathcal{M}_{\mathrm{as}}^{\mu}:=\bigcup_{P \in \underline{\underline{\mathrm{As}}}} \mathcal{M}_{P}^{\mu}
$$

as the space of all Mellin symbols of order $\mu$. Notice that $\mathcal{M}_{P}^{\mu}=\mathcal{M}_{\mathcal{O}}^{\mu}+\mathcal{M}_{P}^{-\infty}$ and $\mathcal{M}_{\text {as }}^{\mu}=\mathcal{M}_{\mathcal{O}}^{\mu}+\mathcal{M}_{\text {as }}^{-\infty}$.

For $h \in \mathcal{M}_{\mathcal{O}}^{\mu}, \mu \in \mathbb{R}$, the principal symbol $\sigma_{\psi}^{\mu}\left(\left.h(z)\right|_{z=\beta+i \tau}\right)$ is independent of $\beta \in \mathbb{R}$. Writing $h=h_{0}+g$ for a general $h \in \mathcal{M}_{\text {as }}^{\mu}, \mu \in \mathbb{R}$, where $h_{0} \in \mathcal{M}_{\mathcal{O}}^{\mu}$, $g \in \mathcal{M}_{\text {as }}^{-\infty}$, we obtain a uniquely defined principal symbol

$$
\sigma_{\psi}^{\mu}(h)(\tau):=\sigma_{\psi}^{\mu}\left(\left.h_{0}(z)\right|_{z=\beta+i \tau}\right) \in S^{(\mu)}\left(\mathbb{R}_{\tau} \backslash 0\right)
$$

Definition 2.5. Let $h \in \mathcal{M}_{\text {as }}^{\mu}, \mu \in \mathbb{R}$. Then $h$ is called elliptic if $\sigma_{\psi}^{\mu}(h)(\tau) \neq 0$ for all $\tau \neq 0$.

Note that, for an elliptic $h \in \mathcal{M}_{\text {as }}^{\mu}$, we have $h^{-1} \in \mathcal{M}_{\text {as }}^{-\mu}$.

From LIU [13, Proposition 3.3], we quote the following result which, in fact, is a corollary of the factorization result of WITT [27]:

Proposition 2.6. For $P \in \underline{A s}, \mu \in \mathbb{R}$, there exists an elliptic $f \in \mathcal{M}_{P}^{\mu}$ such that $f^{-1} \in \mathcal{M}_{\mathcal{O}}^{-\mu}$. Furthermore, in case $\mu=0, f$ can be chosen in the form

$$
f(z)=1+g(z)
$$

where $g \in \mathcal{M}_{P}^{-\infty}$.

To get further control on Mellin symbols, we state, see LIU [13, Proposition 3.5]: 
Proposition 2.7. There is a system of meromorphic functions $g_{P}(z)$ on $\mathbb{C}$, indexed by asymptotic types $P \in \underline{\text { As, }}$ such that

$$
\begin{gathered}
\left(g_{P}(z)\right)=P \quad \text { for all } P \in \underline{\mathrm{As}}, \\
\frac{g_{Q}(z+\mu)}{g_{P}(z)} \in \mathcal{M}_{\mathrm{as}}^{\mu} \quad \text { for all } \mu \in \mathbb{Z}, P, Q \in \underline{\mathrm{As}} .
\end{gathered}
$$

Furthermore, it can be accomplished that the principal symbol $b_{\mu} \in S^{(\mu)}\left(\mathbb{R}_{\tau} \backslash 0\right)$ of the function in (2.4) is independent of $P, Q \in \underline{\text { As }}$ and, in fact, $b_{\mu}(\tau)=$ $b_{1}(\tau)^{\mu}$.

Proof. By virtue of Proposition 2.6, for each $P \in \underline{A s}$, we can choose a $k_{P}(z) \in$ $\mathcal{M}_{\mathcal{P}}^{-\infty}$ such that $\left(1+k_{P}(z)\right)^{-1} \in \mathcal{M}_{\mathcal{O}}^{0}$. Then the functions (see Example 2.2)

$$
g_{P}(z):=\Gamma(z) \frac{1+k_{P}(z)}{1+k_{P_{0}}(z)},
$$

where $\Gamma(z)$ is the $\Gamma$-function (in particular, $(\Gamma(z))=-P_{0}$ and $\frac{\Gamma(z+\mu)}{\Gamma(z)} \in \mathcal{M}_{\text {as }}^{\mu}$ for all $\mu \in \mathbb{Z}$, see OLVER [17]) are as required.

2.3. Function spaces. Cone Sobolev spaces $\mathbb{H}_{P}^{s, \delta}\left(\mathbb{R}_{+}\right)$including asymptotic information encoded by the asymptotic type $P \in \underline{A s} \underline{s}^{\delta}$ are introduced. The length of the closed interval on which asymptotics of type $P$ are observed equals the Sobolev smoothness $s \geq 0$. The spaces $\mathbb{H}_{P}^{s, \delta}\left(\mathbb{R}_{+}\right)$are similar to the spaces $\mathcal{K}_{P, \vartheta-0}^{s, \gamma}\left(\mathbb{R}_{+}\right)$used in Appendix A; with the difference that the spaces now are Hilbert spaces rather than only Fréchet-Hilbert spaces. For the general idea of using spaces with asymptotics, see REMPEL-Schulze [19], SchulzE [21].

Definition 2.8. For $s \geq 0, P \in \underline{A s}^{\delta}$, the space $\mathbb{H}_{P}^{s, \delta}\left(\mathbb{R}_{+}\right)$consists of all $u \in$ $H_{\mathrm{loc}}^{s}\left(\mathbb{R}_{+}\right)$such that $(1-\omega) u \in t^{\delta} H^{s}\left(\mathbb{R}_{+}\right)$and, for $v:=\omega u$, we have $h(z) \tilde{v}(z) \in$ $\mathcal{A}(S(1 / 2-\delta-s, 1 / 2-\delta))$ and

$$
\sup _{s^{\prime} \in(0, s)}\left\{\frac{1}{2 \pi i} \int_{\operatorname{Re} z=1 / 2-\delta-s^{\prime}}|h(z) \tilde{v}(z)|^{2} d z\right\}^{1 / 2}<+\infty
$$

for some (and then any) $h(z) \in \mathcal{M}_{\mathcal{O}}^{s}$ that fulfils $h^{-1}(z) \in \mathcal{M}_{P}^{-s}$. 
Remark 2.9. The assumptions in Definition 2.8 ensure that the limit

$$
\left.h(z) v(z)\right|_{z=1 / 2-\delta-s^{\prime}-i \tau} \rightarrow w(\tau) \quad \text { in } L^{2}\left(\mathbb{R}_{\tau}\right) \text { as } s^{\prime} \rightarrow s
$$

exists. Furthermore, since the support of $v=\omega u$ is bounded, we also have $h(z) \tilde{v}(z) \in \mathcal{A}(S(1 / 2-\delta-s, 1 / 2-\delta+a))$ and $\sup _{s^{\prime} \in(-a, s)}\{\ldots\}^{1 / 2}<+\infty$ instead of (2.5) for any $a>0$.

Example 2.10. For all $s \geq 0, H^{s}\left(\mathbb{R}_{+}\right)=\mathbb{H}_{P_{0}}^{s, 0}\left(\mathbb{R}_{+}\right)$as well as $H_{0}^{s}\left(\overline{\mathbb{R}}_{+}\right)=$ $\mathbb{H}_{\mathcal{O}}^{s, 0}\left(\mathbb{R}_{+}\right)$, where $H_{0}^{s}\left(\overline{\mathbb{R}}_{+}\right)$is the space of all $u \in H^{s}(\mathbb{R})$ having support in $[0, \infty)$.

For $P \in \underline{A s^{\delta}}, s \geq 0$, we set $\mathcal{E}_{P}^{\delta}:=\{1 / 2-\delta-\operatorname{Re} p ; p \in \operatorname{supp} P\}$,

$$
D_{P}^{s}:=\{(p, k) \in S(1 / 2-\delta-s, 1 / 2-\delta) \times \mathbb{N} ; k+1 \leq P(p)\}
$$

Definition 2.11. For $(p, k) \in S(1 / 2-\delta) \times \mathbb{N}$, the trace operator $T(p, k)$ is defined by

$$
T(p, k) u:=\frac{(-1)^{k}}{k !} \operatorname{Res}_{z=p}\left((z-p)^{k} \widetilde{\omega} u(z)\right), \quad u \in \bigcup_{\substack{P \in \mathrm{As}^{\delta}, s>1 / 2-\delta-\operatorname{Re} p}} \mathbb{H}_{P}^{s, \delta}\left(\mathbb{R}_{+}\right) .
$$

Example 2.12. For $(q, l) \in S(1 / 2-\delta) \times \mathbb{N}$,

$$
T(p, k)\left(\omega(t) t^{-q} \log ^{l} t\right)= \begin{cases}1 & \text { if }(q, l)=(p, k), \\ 0 & \text { otherwise }\end{cases}
$$

Now, we list some of the properties of the function spaces $\mathbb{H}_{P}^{s, \delta}\left(\mathbb{R}_{+}\right)$. For proofs and further information, the reader is referred to LIU [13].

Proposition 2.13. (a) $\left\{\mathbb{H}_{P}^{s, \delta}\left(\mathbb{R}_{+}\right) ; s \geq 0\right\}$ forms an interpolation scale of Hilbert spaces with respect to the complex interpolation method.

(b) $\mathbb{H}_{P}^{s, \delta}\left(\mathbb{R}_{+}\right) \subseteq \mathbb{H}_{P}^{s^{\prime}, \delta}\left(\mathbb{R}_{+}\right)$with continuous embedding if and only if $s \geq s^{\prime}$. Furthermore, for any $a>0$, the embedding $\left\{u \in \mathbb{H}_{P}^{s, \delta}\left(\mathbb{R}_{+}\right)\right.$; suppu $\left.\subseteq(0, a]\right\} \subset$ $\mathbb{H}_{P}^{s^{\prime}, \delta}\left(\mathbb{R}_{+}\right)$is compact if and only if $s>s^{\prime}$. 
(c) For $(p, k) \in S(1 / 2-\delta) \times \mathbb{N}$, the trace operator $T(p, k): \mathbb{H}_{P}^{s, \delta}\left(\mathbb{R}_{+}\right) \rightarrow \mathbb{C}$ is well-defined. It is zero if $(p, k) \notin P$. For $(p, k) \in P$, however, the short sequence

$$
0 \longrightarrow \mathbb{H}_{\mathcal{O}}^{s, \delta}\left(\mathbb{R}_{+}\right) \longrightarrow \mathbb{H}_{P}^{s, \delta}\left(\mathbb{R}_{+}\right) \stackrel{(T(p, k))_{(p, k) \in D_{P}^{s}}}{\longrightarrow} \bigoplus_{(p, k) \in D_{P}^{s}} \mathbb{C} \longrightarrow 0
$$

is split exact provided that $s \notin \mathcal{E}_{P}^{\delta}$. In that case, a splitting is provided by the operator

$$
\bigoplus_{(p, k) \in D_{P}^{s}} \mathbb{C} \rightarrow \mathbb{H}_{P}^{s, \delta}\left(\mathbb{R}_{+}\right), \quad\left(c_{p k}\right)_{(p, k) \in D_{P}^{s}} \mapsto \sum_{(p, k) \in D_{P}^{s}} \omega(t) t^{-p} \log ^{k} t c_{p k}
$$

The space $\mathbb{H}^{0, \delta}\left(\mathbb{R}_{+}\right):=\mathbb{H}_{P}^{0, \delta}\left(\mathbb{R}_{+}\right)$is independent of $P$. Its scalar product is given by

$$
\langle u, v\rangle:=\int_{0}^{\infty} u(t) \overline{v(t)} t^{-2 \delta} d t
$$

For $P \in \underline{A s}^{\delta}$, we further introduce

$$
\mathcal{S}_{P}\left(\mathbb{R}_{+}\right):=\left\{u \in C^{\infty}\left(\mathbb{R}_{+}\right) ; \omega u \in \bigcap_{s \geq 0} \mathbb{H}_{P}^{s, \delta}\left(\mathbb{R}_{+}\right),(1-\omega) u \in \mathcal{S}\left(\overline{\mathbb{R}}_{+}\right)\right\}
$$

$\mathcal{S}_{P}\left(\mathbb{R}_{+}\right)$is a nuclear Fréchet space. We will likewise employ the notation

$$
\mathcal{S}_{\mathrm{as}}^{\delta}\left(\mathbb{R}_{+}\right):=\bigcup_{P \in \underline{\mathrm{As}^{\delta}}} \mathcal{S}_{P}\left(\mathbb{R}_{+}\right)
$$

\section{Basic Elements of the Calculus}

In this section, the basic elements of the calculus are introduced. The relation of the calculus to Boutet de Monvel's calculus for pseudodifferential boundary value problems is uncovered in an appendix to Section 3.3. 
3.1. Interior symbols. To begin with, we present the necessary background concerning interior symbols. The basic observation is that upon controlling complete Mellin symbols one can control the mapping properties of the corresponding cone pseudodifferential operators.

Lemma 3.1. Let $h \in \mathcal{M}_{\text {as }}^{\mu}$ satisfy $(h(z)) \succcurlyeq P-T^{-\mu} Q$ for some $P, Q \in \underline{A s^{\sharp}}$. Then, for all $s \geq \mu^{+}$,

$$
\omega(t) t^{-\mu} \mathrm{op}_{M}^{1 / 2-\delta}(h(z)) \omega_{0}(t): \mathbb{H}_{P}^{s, \delta}\left(\mathbb{R}_{+}\right) \rightarrow \mathbb{H}_{Q}^{s-\mu, \delta}\left(\mathbb{R}_{+}\right)
$$

continuously.

Proof. Let $h_{P}^{s} \in \mathcal{M}_{\mathcal{O}}^{s}$ with $\left(h_{P}^{s}\right)^{-1} \in \mathcal{M}_{P}^{-s}$ and $h_{Q}^{s-\mu} \in \mathcal{M}_{\mathcal{O}}^{s-\mu}$ with $\left(h_{Q}^{s-\mu}\right)^{-1} \in$ $\mathcal{M}_{Q}^{-(s-\mu)}$. Then, for $u \in \mathbb{H}_{P}^{s, \delta}\left(\mathbb{R}_{+}\right), v:=\omega_{0} u$, we have $h_{P}^{s}(z) \tilde{v}(z) \in \mathcal{A}(S(1 / 2-$ $\delta-s, 1 / 2-\delta-\mu))$,

$$
\sup _{s^{\prime} \in(-\mu, s)}\left\|h_{P}^{s}(z) \tilde{v}(z)\right\|_{L^{2}\left(\Gamma_{1 / 2-\delta-s^{\prime}}\right)}<\infty
$$

Moreover,

$$
\begin{aligned}
h_{Q}^{s-\mu}(z) M_{t \rightarrow z}\left\{t^{-\mu} \mathrm{op}_{M}^{1 / 2-\delta}(h) v\right\} & \\
& =h_{Q}^{s-\mu}(z) h(z-\mu) h_{P}^{s}(z-\mu)^{-1} h_{P}^{s}(z-\mu) \tilde{v}(z-\mu),
\end{aligned}
$$

where

$$
h_{Q}^{s-\mu}(z) h(z-\mu) h_{P}^{s}(z-\mu)^{-1} \in \mathcal{M}_{\mathcal{O}}^{0} .
$$

This yields

$$
\sup _{s^{\prime} \in(0, s-\mu)}\left\|h_{Q}^{s-\mu}(z) M_{t \rightarrow z}\left\{t^{-\mu} \operatorname{op}_{M}^{1 / 2-\delta}(h) v\right\}\right\|_{L^{2}\left(\Gamma_{1 / 2-\delta-s^{\prime}}\right)}<\infty
$$

finishing the proof.

Remark 3.2. The proof of Lemma 3.1 shows that in case $\mu<0$

$$
T(q, l)\left(\omega t^{-\mu} \mathrm{op}_{M}^{1 / 2-\delta}(h) \omega_{0} u\right)=0
$$

if $u \in \mathbb{H}_{P}^{s, \delta}\left(\mathbb{R}_{+}\right),(q, l) \in D_{Q}^{-\mu}$. 
Definition 3.3. Let $\mu \in \mathbb{Z}, P, Q \in \underline{A s^{\sharp}}$.

(i) The class $S_{\mathrm{cl}}^{\mu}\left(\overline{\mathbb{R}}_{+} \times \Gamma_{1 / 2-\delta}\right)_{P, Q}$ consists of all $h(t, z) \in S_{\mathrm{cl}}^{\mu}\left(\overline{\mathbb{R}}_{+} \times \Gamma_{1 / 2-\delta}\right)$ such that $\partial_{t}^{j} h(0, z) \in \mathcal{M}_{\text {as }}^{\mu}$,

$$
\left(\partial_{t}^{j} h(0, z)\right) \succcurlyeq P-T^{-\mu+j} Q
$$

for all $j \in \mathbb{N}$.

(ii) The class $S_{\text {cl }}^{\mu}\left(\overline{\mathbb{R}}_{+} \times \Gamma_{1 / 2-\delta}\right)_{0}$ consists of all $h(t, z) \in S_{\text {cl }}^{\mu}\left(\overline{\mathbb{R}}_{+} \times \Gamma_{1 / 2-\delta}\right)$ such that $\partial_{t}^{j} h(0, z)=0$ for all $j \in \mathbb{N}$.

$S_{\mathrm{cl}}^{\mu}\left(\overline{\mathbb{R}}_{+} \times \Gamma_{1 / 2-\delta}\right)_{P, Q}, S_{\mathrm{cl}}^{\mu}\left(\overline{\mathbb{R}}_{+} \times \Gamma_{1 / 2-\delta}\right)_{0}$ are nuclear Fréchet spaces in a natural manner.

Lemma 3.4. Let $g_{P}(z), g_{Q}(z)$ be as in Proposition 2.7. Then (3.1) is equivalent to

$$
\partial_{t}^{j} h(0, z)=a_{j}(z) \frac{g_{Q}(z+\mu-j)}{g_{P}(z)}
$$

for a certain $a_{j} \in \mathcal{M}_{\mathcal{O}}^{j}$.

Remark 3.5. In LiU [13],

$$
\partial_{t}^{j} h(0, z)=a_{j} \frac{g_{P}(z+\mu-j)}{g_{P}(z)}, \quad a_{j} \in \mathbb{C},
$$

for $j=0,1,2, \ldots$ and a certain $P \in \underline{A s}^{\sharp}$ has been assumed.

In the classes $S_{\mathrm{cl}}^{\mu}\left(\overline{\mathbb{R}}_{+} \times \Gamma_{1 / 2-\delta}\right)_{P, Q}$, asymptotic summation is possible:

Proposition 3.6. Let $\left\{\mu_{j}\right\}_{j \in \mathbb{N}} \subset \mathbb{Z}$ be a sequence such that $\mu_{j} \rightarrow-\infty$ as $j \rightarrow$ $\infty$. Let $h_{j} \in S_{\mathrm{cl}}^{\mu_{j}}\left(\overline{\mathbb{R}}_{+} \times \Gamma_{1 / 2-\delta}\right)_{P, Q}$ for $j=0,1,2, \ldots$ and $\bar{\mu}=\max \left\{\mu_{j} ; j \in \mathbb{N}\right\}$. Then, for $c_{j} \rightarrow \infty$ as $j \rightarrow \infty$ sufficiently fast, the infinite sum

$$
h^{\prime}(t, z):=\sum_{j=0}^{\infty} \omega\left(c_{j} t\right) t^{\bar{\mu}-\mu_{j}} h_{j}(t, z)
$$

belongs to $S_{\mathrm{cl}}^{\bar{\mu}}\left(\overline{\mathbb{R}}_{+} \times \Gamma_{1 / 2-\delta}\right)_{P, Q}$. 
Proof. For each $t \geq 0$, the sum on the right-hand side of (3.3) is finite. Therefore, $h^{\prime}(t, z)$ is well-defined. Thus, it suffices to prove that the sequence $\left\{\sum_{j=0}^{l} \omega\left(c_{j} t\right) t^{\bar{\mu}-\mu_{j}} h_{j}(t, z)\right\}_{l \in \mathbb{N}}$ is bounded in $S_{\mathrm{cl}}^{\bar{\mu}}\left(\overline{\mathbb{R}}_{+} \times \Gamma_{1 / 2-\delta}\right)_{P, Q}$ (see Remark 3.9 below) when the sequence $\left\{c_{j}\right\} \subset \mathbb{R}_{+}$is appropriately chosen.

Let $\alpha_{j m k T}:=\sup _{0 \leq t \leq T} \sup _{m^{\prime} \leq m} \sup _{z \in \Gamma_{1 / 2-\delta}}\langle z\rangle^{-\bar{\mu}+k}\left|\partial_{t}^{m^{\prime}} \partial_{z}^{k} h_{j}(t, z)\right|$ and choose, by a diagonal trick, $c_{j} \rightarrow \infty$ as $j \rightarrow \infty$ so fast that, for all $T>0, m, k \in \mathbb{N}$,

$$
\sup _{0 \leq t \leq T} \sup _{m^{\prime} \leq m} \sum_{j=0}^{\infty}\left|\partial_{t}^{m-m^{\prime}}\left(\omega\left(c_{j} t\right) t^{\bar{\mu}-\mu_{j}}\right)\right| \alpha_{j m k T}<\infty \text {. }
$$

Then, for all $T>0, m, k \in \mathbb{N}$, and some constants $C(T, m, k)>0$ independently of $l$,

$$
\sup _{0 \leq t \leq T} \sup _{z \in \Gamma_{1 / 2-\delta}}\langle z\rangle^{-\bar{\mu}+k}\left|\sum_{j=0}^{l} \partial_{t}^{m} \partial_{z}^{k}\left(\omega\left(c_{j} t\right) t^{\bar{\mu}-\mu_{j}} h_{j}(t, z)\right)\right| \leq C(T, m, k) .
$$

This shows boundedness of the sequence $\left\{\sum_{j=0}^{l} \omega\left(c_{j} t\right) t^{\bar{\mu}-\mu_{j}} h_{j}(t, z)\right\}_{l \in \mathbb{N}}$ in the space $S^{\bar{\mu}}\left(\overline{\mathbb{R}}_{+} \times \Gamma_{1 / 2-\delta}\right)$.

Upon subtracting leading homogeneous components and repeating this procedure, we finally arrive at boundedness in $S_{\mathrm{cl}}^{\bar{\mu}}\left(\overline{\mathbb{R}}_{+} \times \Gamma_{1 / 2-\delta}\right)_{P, Q}$.

Proposition 3.7. For all $s \geq \mu^{+}$, the linear mapping

$$
S_{\mathrm{cl}}^{\mu}\left(\overline{\mathbb{R}}_{+} \times \Gamma_{1 / 2-\delta}\right)_{P, Q} \rightarrow \mathcal{L}\left(\mathbb{H}_{P}^{s, \delta}\left(\mathbb{R}_{+}\right), \mathbb{H}_{Q}^{s-\mu, \delta}\left(\mathbb{R}_{+}\right)\right), \quad h \mapsto \omega t^{-\mu} \mathrm{op}_{M}^{1 / 2-\delta}(h) \omega_{0}
$$

is well-defined and continuous.

Proof. Here, the main point is to prove that $\omega t^{-\mu} \mathrm{op}_{M}^{1 / 2-\delta}(h) \omega_{0} u \in \mathbb{H}_{Q}^{s-\mu, \delta}\left(\mathbb{R}_{+}\right)$ for all $u \in \mathbb{H}_{P}^{s, \delta}\left(\mathbb{R}_{+}\right)$. Let $h_{j}(z):=\frac{1}{j !} \partial_{t}^{j} h(0, z)$. Upon choosing $c_{j} \rightarrow \infty$ as $j \rightarrow \infty$ sufficiently fast (in dependence on $u$, but at least as fast as in Proposition 3.6), we may arrange that

$$
\left\|\omega\left(c_{j} t\right) t^{-\mu+j} \operatorname{op}_{M}^{1 / 2-\delta}\left(h_{j}\right) \omega_{0} u\right\|_{\mathbb{H}_{Q}^{s-\mu, \delta}\left(\mathbb{R}_{+}\right)} \leq 2^{-j}
$$


for all $j$. But then the series

$$
\sum_{j=0}^{\infty} \omega t^{-\mu} \operatorname{op}_{M}^{1 / 2-\delta}\left(\omega\left(c_{j} t\right) t^{j} h_{j}\right) \omega_{0} u
$$

converges absolutely in $\mathbb{H}_{Q}^{s-\mu, \delta}\left(\mathbb{R}_{+}\right)$. Let $h^{\prime}(t, z)$ as in Proposition 3.6. Then, $h(t, z)-h^{\prime}(t, z) \in S_{\mathrm{cl}}^{\mu}\left(\overline{\mathbb{R}}_{+} \times \Gamma_{1 / 2-\delta}\right)_{0}$ and, obviously,

$$
\omega(t) t^{-\mu} \operatorname{op}_{M}^{1 / 2-\delta}\left(h-h^{\prime}\right) \omega_{0}(t): \mathbb{H}_{P}^{s, \delta}\left(\mathbb{R}_{+}\right) \rightarrow \mathbb{H}_{\mathcal{O}}^{s-\mu, \delta}\left(\mathbb{R}_{+}\right) .
$$

The proof is concluded by appealing to the closed graph theorem twice.

For the sake of completeness, we mention:

Proposition 3.8. Let $\left\{h_{l}(t, z)\right\}_{l=0}^{\infty} \subset S_{\mathrm{cl}}^{\mu}\left(\overline{\mathbb{R}}_{+} \times \Gamma_{1 / 2-\delta}\right)_{P, Q}$ be a sequence such that

$$
h_{l}(t, z) \rightarrow h(t, z) \text { in } S_{\mathrm{cl}}^{\mu}\left(\overline{\mathbb{R}}_{+} \times \Gamma_{1 / 2-\delta}\right)_{P, Q} \text { as } l \rightarrow \infty
$$

Then, for all $s \geq \mu^{+}$,

$$
\omega(t) t^{-\mu} \mathrm{op}_{M}^{1 / 2-\delta}\left(h_{l}\right) \omega_{0}(t) \rightarrow \omega(t) t^{-\mu} \mathrm{op}_{M}^{1 / 2-\delta}(h) \omega_{0}(t)
$$

strongly in $\mathcal{L}\left(\mathbb{H}_{P}^{s, \delta}\left(\mathbb{R}_{+}\right), \mathbb{H}_{Q}^{s-\mu, \delta}\left(\mathbb{R}_{+}\right)\right)$as $l \rightarrow \infty$.

Remark 3.9. Let $\left\{h_{l}(t, z)\right\}_{l=0}^{\infty}$ be a bounded sequence in $S_{\mathrm{cl}}^{\mu}\left(\overline{\mathbb{R}}_{+} \times \Gamma_{1 / 2-\delta}\right)_{P, Q}$ that converges point-wise to some $h(t, z)$. Then $h(t, z) \in S_{\mathrm{cl}}^{\mu}\left(\overline{\mathbb{R}}_{+} \times \Gamma_{1 / 2-\delta}\right)_{P, Q}$ and (3.4) holds.

3.2. Green, trace, and potential operators. The calculus is now completed by trace and potential operators. At the same time, the residual Green operators are introduced. This is also here that types $d \in \mathbb{N}$ occur for the first time.

For $P \in \underline{A s^{\sharp}}$, let $d_{0}=\min \left\{d \in \mathbb{N} ; D_{P}^{d} \neq \emptyset\right\}$ and then, for $d \geq d_{0}$,

$$
s_{d}:=\max \left\{1 / 2-\delta-\operatorname{Re} p ;(p, k) \in D_{P}^{d}\right\} .
$$


Moreover, we introduce

$$
\zeta_{P}^{d} u(t):=u(t)-\sum_{(p, k) \in D_{P}^{d}} \omega(t) t^{-p} \log ^{k} t T(p, k) u
$$

for any $d \in \mathbb{N}$, where $u \in \bigcup_{s>s_{d}} \mathbb{H}_{P}^{s, \delta}\left(\mathbb{R}_{+}\right)$when $d \geq d_{0}$, and $u \in \mathbb{H}^{0, \delta}\left(\mathbb{R}_{+}\right)$ otherwise.

Definition 3.10. For $d, N_{-}, N_{+} \in \mathbb{N}$, the class $\Psi_{G ; P, Q}^{d}\left(\mathbb{R}_{+} ; N_{-}, N_{+}\right)$consists of all operator block matrices

$$
\mathcal{G}=\left(\begin{array}{ll}
G & K \\
T & Q
\end{array}\right): \begin{array}{cccc}
\mathcal{S}_{P}\left(\mathbb{R}_{+}\right) & \multicolumn{1}{c}{\mathcal{S}_{Q}\left(\mathbb{R}_{+}\right)} \\
& \mathbb{C}^{N_{-}} & & \mathbb{C}^{N_{+}}
\end{array},
$$

where

$$
G u=\operatorname{op}_{G}(g) \zeta_{P}^{d} u+\sum_{(p, k) \in D_{P}^{d}} d_{p k}(t) T(p, k) u
$$

for certain $g \in \mathcal{S}_{Q}\left(\mathbb{R}_{+}\right) \hat{\otimes}_{\pi} \mathcal{S}_{\text {as }}^{\delta}\left(\mathbb{R}_{+}\right), d_{p k} \in \mathcal{S}_{Q}\left(\mathbb{R}_{+}\right), T=\left(T_{1}, \ldots, T_{N_{+}}\right)$,

$$
T_{j} u=\left\langle\zeta_{P}^{d} u, b_{j}\right\rangle+\sum_{(p, k) \in D_{P}^{d}} \beta_{j p k} T(p, k) u
$$

for $j=1, \ldots, N_{+}$and certain $b_{j} \in \mathcal{S}_{\mathrm{as}}^{\delta}\left(\mathbb{R}_{+}\right), \beta_{j p k} \in \mathbb{C}$,

$$
K c=\sum_{l=1}^{N_{-}} c_{l} k_{l}(t), \quad c=\left(c_{1}, \ldots, c_{N_{-}}\right) \in \mathbb{C}^{N_{-}},
$$

for certain $k_{l} \in \mathcal{S}_{Q}\left(\mathbb{R}_{+}\right)$, and $Q$ is a $N_{+} \times N_{-}$matrix.

In (3.7), $G$ is called a Green operator of type $d, T$ is called a trace operator of type $d$, and $K$ is called a potential operator. We also write $\Psi_{G ; P, Q}^{d}\left(\mathbb{R}_{+}\right):=$ $\Psi_{G ; P, Q}^{d}\left(\mathbb{R}_{+} ; 0,0\right)$ and $\Psi_{G ; P}^{d}\left(\mathbb{R}_{+} ; N_{-}, N_{+}\right):=\Psi_{G ; P, P}^{d}\left(\mathbb{R}_{+} ; N_{-}, N_{+}\right)$. 
Proposition 3.11. For all $s>s_{d}$ when $d \geq d_{0}$, and $s \geq 0$ otherwise, the operator $\mathcal{G}$ from $(3.7)$ extends to a continuous operator

$$
\begin{aligned}
& \mathbb{H}_{P}^{s, \delta}\left(\mathbb{R}_{+}\right) \quad \mathcal{S}_{Q}\left(\mathbb{R}_{+}\right) \\
& \mathcal{G}: \oplus \quad \rightarrow \quad \oplus \text {. } \\
& \mathbb{C}^{N_{-}} \quad \mathbb{C}^{N_{+}}
\end{aligned}
$$

3.3. Definition, mapping properties. Now, we are in a position to define the basic operator classes.

Definition 3.12. (i) For $\mu \in \mathbb{Z}, d \in \mathbb{N}, P, Q \in \underline{\text {As}^{\sharp}}$, the class $\Psi_{P, Q}^{\mu, d}\left(\mathbb{R}_{+}\right)$ consists of all operators $A: \mathcal{S}_{P}\left(\mathbb{R}_{+}\right) \rightarrow \mathcal{S}_{Q}\left(\mathbb{R}_{+}\right)$of the form

$$
A=\omega(t) t^{-\mu} \operatorname{op}_{M}^{1 / 2-\delta}(h(t, z)) \omega_{0}(t)+(1-\omega(t)) \operatorname{op}_{\psi}(p)\left(1-\omega_{1}(t)\right)+G,
$$

where $h \in S_{\text {cl }}^{\mu}\left(\overline{\mathbb{R}}_{+} \times \Gamma_{1 / 2-\delta}\right)_{P, Q}, p \in S_{\text {cl }}^{\mu}\left(\mathbb{R}_{+} \times \mathbb{R}\right) \cap S^{\mu ; 0}\left(\mathbb{R}_{+} \times \mathbb{R}\right)$, and $G \in$ $\Psi_{G ; P, Q}^{d}\left(\mathbb{R}_{+}\right)$.

(ii) For $\mu \in \mathbb{Z}, d, N_{-}, N_{+} \in \mathbb{N}, P, Q \in \underline{\mathrm{As}^{\sharp}}$, the class $\Psi_{P, Q}^{\mu, d}\left(\mathbb{R}_{+} ; N_{-}, N_{+}\right)$ consists of all operator block matrices

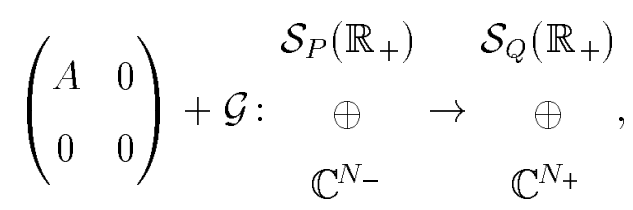

where $A \in \Psi_{P, Q}^{\mu, d}\left(\mathbb{R}_{+}\right)$and $\mathcal{G} \in \Psi_{G ; P, Q}^{d}\left(\mathbb{R}_{+} ; N_{-}, N_{+}\right)$.

In case we have to mention the dependence of these operator classes on $\delta$ explicitly (e.g., in Section 3.4), we write $\Psi_{P, Q}^{\mu, d ; \delta}\left(\mathbb{R}_{+} ; N_{-}, N_{+}\right)$etc. As before, we also write $\Psi_{P}^{\mu, d}\left(\mathbb{R}_{+} ; N_{-}, N_{+}\right)$in place of $\Psi_{P, P}^{\mu, d}\left(\mathbb{R}_{+} ; N_{-}, N_{+}\right)$.

Remark 3.13. In (3.8), we may always assume that

$$
t^{-\mu} \mathrm{op}_{M}^{1 / 2-\delta}(h(t, z))-\mathrm{op}_{\psi}(p) \in L^{-\infty}\left(\mathbb{R}_{+}\right) .
$$

For this kind of argument, see ScHulze [22, 23]

Proof of Theorem 1.1. The proof easily follows from Propositions 3.7, 3.11. 
We conclude this section with a couple of remarks.

Lemma 3.14. Let $h \in S^{-\infty}\left(\overline{\mathbb{R}}_{+} \times \Gamma_{1 / 2-\delta}\right)_{P, Q}, p \in S^{-\infty ;-\infty}\left(\mathbb{R}_{+} \times \mathbb{R}\right)$. Then the operator $A$ in $(3.8)$ belongs to $\Psi_{G ; P, Q}^{d}\left(\mathbb{R}_{+}\right)$.

Asymptotic summation is again possible:

Proposition 3.15. Let $\left\{\mu_{j}\right\} \subset \mathbb{Z}$ be a sequence such that $\mu_{j} \rightarrow-\infty$ as $j \rightarrow$ $\infty$. Let $\mathcal{A}_{j} \in \Psi_{P, Q}^{\mu_{j}, d}\left(\mathbb{R}_{+} ; N_{-}, N_{+}\right)$for $j=0,1,2, \ldots$ Then there is an $\mathcal{A} \in$ $\Psi_{P, Q}^{\bar{\mu}, d}\left(\mathbb{R}_{+} ; N_{-}, N_{+}\right)$, where $\bar{\mu}=\max \left\{\mu_{j} ; j \in \mathbb{N}\right\}$, such that, for all $J \in \mathbb{N}$,

$$
\mathcal{A}-\sum_{j<J} \mathcal{A}_{j} \in \Psi_{P, Q}^{\bar{\mu}_{J}, d}\left(\mathbb{R}_{+} ; N_{-}, N_{+}\right)
$$

where $\bar{\mu}_{j}=\max \left\{\mu_{j} ; j \geq J\right\}$. Moreover, $\mathcal{A}$ is uniquely determined modulo $\Psi_{P, Q}^{-\infty, d}\left(\mathbb{R}_{+} ; N_{-}, N_{+}\right)$.

Proof. We may restrict to the case $N_{-}=N_{+}=0$. Let $A_{j} \in \Psi_{P, Q}^{\mu_{j}, d}\left(\mathbb{R}_{+}\right)$for $j=0,1,2, \ldots$, where

$$
A_{j}=\omega t^{-\mu_{j}} \mathrm{op}_{M}^{1 / 2-\delta}\left(h_{j}\right) \omega_{0}+(1-\omega) \mathrm{op}_{\psi}\left(p_{j}\right)\left(1-\omega_{1}\right)+G_{j},
$$

$h_{j} \in S_{\mathrm{cl}}^{\mu_{j}}\left(\overline{\mathbb{R}}_{+} \times \Gamma_{1 / 2-\delta}\right)_{P, Q}, p_{j} \in S_{\mathrm{cl}}^{\mu_{j}}\left(\mathbb{R}_{+} \times \mathbb{R}\right) \cap S^{\mu_{j} ; 0}\left(\mathbb{R}_{+} \times \mathbb{R}\right)$, and $G_{j} \in$ $\Psi_{G ; P, Q}^{d}\left(\mathbb{R}_{+}\right)$. Choose $h \in S_{\mathrm{cl}}^{\bar{\mu}}\left(\overline{\mathbb{R}}_{+} \times \Gamma_{1 / 2-\delta}\right)_{P, Q}, p \in S_{\mathrm{cl}}^{\bar{\mu}}\left(\mathbb{R}_{+} \times \mathbb{R}\right) \cap S^{\bar{\mu} ; 0}\left(\mathbb{R}_{+} \times \mathbb{R}\right)$ such that, for all $J \in \mathbb{N}$,

$$
h-\sum_{j<J} t^{\bar{\mu}-\mu_{j}} h_{j} \in S_{\mathrm{cl}}^{\bar{\mu}_{J}}\left(\overline{\mathbb{R}}_{+} \times \Gamma_{1 / 2-\delta}\right)_{P, Q},
$$

$p-\sum_{j<J} p_{j} \in S_{\mathrm{cl}}^{\bar{\mu}_{J}}\left(\mathbb{R}_{+} \times \mathbb{R}\right) \cap S^{\bar{\mu}_{J} ; 0}\left(\mathbb{R}_{+} \times \mathbb{R}\right)$. For instance, the existence of $h$ in (3.10) is implied by Proposition 3.6. Then it suffices to set $A:=$ $\omega t^{-\bar{\mu}} \mathrm{op}_{M}^{1 / 2-\delta}(h) \omega_{0}+(1-\omega) \mathrm{op}_{\psi}(p)\left(1-\omega_{1}\right)$. 
Appendix to Section 3.3: Boutet de Monvel's calculus. Boutet de Monvel's calculus $B^{\mu, d}\left(\mathbb{R}_{+} ; N_{-}, N_{+}\right)$for pseudodifferential boundary problems, see, e.g., Boutet DE Monvel [1], Schulze [23], appears as a special case of $\Psi_{P, Q}^{\mu, d}\left(\mathbb{R}_{+} ; N_{-}, N_{+}\right)$when $P=Q=P_{0}$, see Example 2.2. Here, the meaning of parameters is the same as before; $\mu \in \mathbb{Z}$ is the order of operators, while $d \in \mathbb{N}$ is their type.

Theorem 3.16. For $\mu \in \mathbb{Z}, d, N_{-}, N_{+} \in \mathbb{N}$, the class $B^{\mu, d}\left(\mathbb{R}_{+} ; N_{-}, N_{+}\right)$consists of all $\mathcal{A} \in \Psi_{P_{0}}^{\mu, d}\left(\mathbb{R}_{+} ; N_{-}, N_{+}\right)$for which, for all $j \in \mathbb{N}$,

$$
\sigma_{M}^{\mu-j}(\mathcal{A})(z)=a_{j}(z) \frac{\Gamma(z+\mu-j)}{\Gamma(z)}
$$

and $a_{j}(z)$ is a polynomial of degree $j$ in $z$.

Proof. From the results of ReMPEL-Schulze [19], we infer that

$$
B^{\mu, 0}\left(\mathbb{R}_{+} ; N_{-}, N_{+}\right) \subset C^{\mu}\left(\mathbb{R}_{+} ; 0,-\mu^{+}\right)
$$

Furthermore, in REMPEL-Schulze [19], also the relation (3.11) has been shown to hold. The proof is completed by noting that the $\Gamma$-function $\Gamma(z)$ can be taken for $g_{P_{0}}(z)$, see $(2.3),(2.4)$, and that $\mathcal{S}_{P_{0}}\left(\mathbb{R}_{+}\right)=\mathcal{S}\left(\overline{\mathbb{R}}_{+}\right)$holds, and upon appealing to Proposition 3.20 below.

Remark 3.17. Note that $a_{0} \in \mathbb{C}$ is just a constant and the ellipticity condition (i) from Definition 4.2 reduces to $a_{0} \neq 0$. See also Remark 4.11.

3.4. Compositions. The proof of Theorem 1.2 is provided in several steps.

Proposition 3.18. Let $\mathcal{A} \in \Psi_{G ; \tilde{Q}, Q}^{d}\left(\mathbb{R}_{+} ; \tilde{N}, N_{+}\right)$or $\mathcal{B} \in \Psi_{G ; P, \tilde{Q}}^{d^{\prime}}\left(\mathbb{R}_{+} ; N_{-}, \tilde{N}\right)$ in Theorem 1.2. Then $\mathcal{A B} \in \Psi_{G ; P, Q}^{d^{\prime \prime}}\left(\mathbb{R}_{+} ; N_{-}, N_{+}\right)$, where $d^{\prime \prime}=\max \left\{d+\mu^{\prime}, d^{\prime}\right\}$ in the first case, while $d^{\prime \prime}=d^{\prime}$ in second one.

Proof. The proof relies on the explicit description of the residual classes in Definition 3.10. It is established by an inspection of all the resulting causes. 
Next, we reduce to the case $\delta=0$. (Note that $\delta \in \mathbb{R}$ has been fixed throughout. After reduction, this fixed $\delta$ is set to 0 .)

Lemma 3.19. For all operators $A$, we have $A \in \Psi_{P, Q}^{\mu, d ; \delta}\left(\mathbb{R}_{+} ; N_{-}, N_{+}\right)$if and only if $t^{-\delta} A t^{\delta} \in \Psi_{T^{\delta} P, T^{\delta} Q}^{\mu, j ; 0}\left(\mathbb{R}_{+} ; N_{-}, N_{+}\right)$, where $T^{\delta} P, T^{\delta} Q \in \underline{A s^{\sharp ; 0}}$ and the space $\Psi_{T^{\delta} P, T^{\delta} Q}^{\mu, d ; 0}\left(\mathbb{R}_{+} ; N_{-}, N_{+}\right)$is assigned to the conormal order 0 .

Proposition 3.20. For $\mu, \mu^{\prime} \in \mathbb{Z}, \mu^{\prime} \geq \mu^{+}$, and $P, Q \in \underline{\mathrm{As}^{\sharp ; 0}}, \Psi_{P, Q}^{\mu, 0 ; 0}\left(\mathbb{R}_{+}\right)$is the class of all operators $A \in C^{\mu}\left(\mathbb{R}_{+} ; 0,-\mu^{\prime}\right)$ (see (A.2)) satisfying

$$
\left(\sigma_{M}^{\mu-j}(A)\right) \succcurlyeq P-T^{-\mu+j} Q, \quad j=0,1,2, \ldots
$$

and, in addition, $A: \mathcal{S}_{P}\left(\mathbb{R}_{+}\right) \rightarrow \mathcal{S}_{Q}\left(\mathbb{R}_{+}\right)$.

Proof. For $A \in \Psi_{P, Q}^{\mu, 0 ; 0}\left(\mathbb{R}_{+}\right)$, it is readily seen that $A \in C^{\mu}\left(\mathbb{R}_{+} ; 0,-\mu^{+}\right)$. Furthermore, we have $C^{\mu}\left(\mathbb{R}_{+} ; 0,-\mu^{+}\right) \subseteq C^{\mu}\left(\mathbb{R}_{+} ; 0,-\mu^{\prime}\right)$ when $\mu^{\prime} \geq \mu^{+}$.

Thus, it remains to prove that each operator $A \in C^{\mu}\left(\mathbb{R}_{+} ; 0,-\mu^{\prime}\right)$ that satisfies the above-mentioned conditions belongs to $\Psi_{P, Q}^{\mu, 0 ; 0}\left(\mathbb{R}_{+}\right)$. Given such an $A$, by virtue of Proposition 3.6, there is an $\tilde{A} \in \Psi_{P, Q}^{\mu, 0 ; 0}\left(\mathbb{R}_{+}\right)$such that $\sigma_{M}^{\mu-j}(\tilde{A})=$ $\sigma_{M}^{\mu-j}(A)$ for all $j=0,1,2, \ldots$ Then $A-\tilde{A} \in C^{\mu}\left(\mathbb{R}_{+} ; 0,-\mu^{\prime}\right)$ satisfies $\sigma_{M}^{\mu-j}(A-$ $\tilde{A})=0$ for $j=0,1,2, \ldots$ and $A-\tilde{A}: \mathcal{S}_{P}\left(\mathbb{R}_{+}\right) \rightarrow \mathcal{S}_{Q}\left(\mathbb{R}_{+}\right)$. Therefore,

$$
A-\tilde{A}=\omega t^{-\mu} \operatorname{op}_{M}^{1 / 2-\delta}(\tilde{h}) \omega_{0}+(1-\omega) \tilde{A}_{\psi}\left(1-\omega_{1}\right)+\tilde{G}
$$

where $\tilde{h} \in S_{\text {cl }}^{\mu}\left(\overline{\mathbb{R}}_{+} \times \Gamma_{1 / 2-\delta}\right)_{0}, \tilde{A}_{\psi} \in L_{\text {cl }}^{\mu}\left(\mathbb{R}_{+}\right) \cap L^{\mu ; 0}\left(\mathbb{R}_{+}\right)$, and $\tilde{G} \in C_{G}\left(\mathbb{R}_{+} ; 0,-\mu^{\prime}\right)$. Since $\omega t^{-\mu} \mathrm{op}_{M}^{1 / 2-\delta}(\tilde{h}) \omega_{0}+(1-\omega) \tilde{A}_{\psi}\left(1-\omega_{1}\right) \in \Psi_{P, Q}^{\mu, 0 ; 0}\left(\mathbb{R}_{+}\right)$, it suffices to show that $\tilde{G} \in \Psi_{G ; P, Q}^{0 ; 0}\left(\mathbb{R}_{+}\right)$.

But $\tilde{G}=\operatorname{op}_{G}(\tilde{g})$ for some $\tilde{g} \in \mathcal{S}_{\mathrm{as}}^{-\mu^{\prime}}\left(\mathbb{R}_{+}\right) \hat{\otimes}_{\pi} \mathcal{S}_{\mathrm{as}}^{0}\left(\mathbb{R}_{+}\right)$(see REMPEL-SCHulzE [19] and also LiU [13, Proposition 5.4]). Then $\tilde{G}: \mathcal{S}_{P}\left(\mathbb{R}_{+}\right) \rightarrow \mathcal{S}_{Q}\left(\mathbb{R}_{+}\right)$implies that, in fact, $\tilde{g} \in \mathcal{S}_{Q}\left(\mathbb{R}_{+}\right) \hat{\otimes}_{\pi} \mathcal{S}_{\text {as }}^{0}\left(\mathbb{R}_{+}\right)$and $\tilde{G} \in \Psi_{G ; P, Q}^{0 ; 0}\left(\mathbb{R}_{+}\right)$.

Proof of Theorem 1.D. By virtue of Lemma 3.19, we may assume that $\delta=0$. Moreover, by Proposition 3.18 , it suffices to treat the case $A:=\mathcal{A} \in \Psi_{\tilde{Q}, Q}^{m, 0 ; 0}\left(\mathbb{R}_{+}\right)$ 
and $B:=\mathcal{B} \in \Psi_{P, \tilde{Q}}^{m^{\prime}, 0 ; 0}\left(\mathbb{R}_{+}\right)$. Then we can employ the characterization from Proposition 3.20 and the composition result in Proposition A.3 for Schulze's cone algebra.

Let us first assume that $\mu^{\prime} \leq 0$. Then $A B \in C^{\mu+\mu^{\prime}}\left(\mathbb{R}_{+} ; 0,-\mu^{+}\right)$,

$$
\begin{aligned}
\left(\sigma_{M}^{\mu+\mu^{\prime}-l}(A B)\right) & \succcurlyeq \bigvee_{j+k=l}\left(\left(T^{\mu^{\prime}-k} \sigma_{M}^{\mu-j}(A)\right)+\left(\sigma_{M}^{\mu^{\prime}-k}(B)\right)\right) \\
& \succcurlyeq \bigvee_{j+k=l}\left(T^{-\mu^{\prime}+k} \tilde{Q}-T^{-\mu^{\prime}+k} T^{-\mu+j} Q+P-T^{-\mu^{\prime}+k} \tilde{Q}\right) \\
& =P-T^{-\mu-\mu^{\prime}+l} Q
\end{aligned}
$$

for all $l=0,1,2, \ldots$ (where, " $\bigvee$ " is join in $(\underline{\mathrm{As}}, \preccurlyeq)$ ), and $A B: \mathcal{S}_{P}\left(\mathbb{R}_{+}\right) \rightarrow$ $\mathcal{S}_{Q}\left(\mathbb{R}_{+}\right)$. This shows that $A B \in \Psi_{P, Q}^{\mu+\mu^{\prime}, 0 ; 0}\left(\mathbb{R}_{+}\right)$in this case.

Now consider $\mu^{\prime}>0$. We write $B=B \zeta_{P}^{\mu^{\prime}}+B\left(1-\zeta_{P}^{\mu^{\prime}}\right)$. Then $B\left(1-\zeta_{P}^{\mu^{\prime}}\right) \epsilon$ $\Psi_{G ; P, \tilde{Q}}^{\mu^{\prime} ; 0}\left(\mathbb{R}_{+}\right)$, while, for a certain $B^{\prime} \in C^{\mu^{\prime}}\left(\mathbb{R}_{+} ; \mu^{\prime}, 0\right), B^{\prime} u=B \zeta_{P}^{\mu^{\prime}} u$ for all $u \in \bigcup_{s>s_{\mu^{\prime}}} \mathbb{H}_{P}^{s, 0}\left(\mathbb{R}_{+}\right)$. This second step corresponds to a shift of the basic line of integration from $\Gamma_{1 / 2-\delta}$ to $\Gamma_{1 / 2-\delta-\mu^{\prime}}$, see (1.5). We obtain $A B^{\prime} \in$ $C^{\mu+\mu^{\prime}}\left(\mathbb{R}_{+} ; \mu^{\prime},-\mu^{+}\right)$and $\left(\sigma_{M}^{\mu+\mu^{\prime}-l}\left(A B^{\prime}\right)\right) \succcurlyeq P-T^{-\mu-\mu^{\prime}+l} Q$ for all $l=0,1,2, \ldots$ as before. Changing back the basic line of integration, we further obtain that $A B^{\prime} u=C u+G u$ for all $u \in \bigcup_{s>s_{\mu^{\prime}}} \mathbb{H}_{P}^{s, 0}\left(\mathbb{R}_{+}\right)$, where $C \in C^{\mu+\mu^{\prime}}\left(\mathbb{R}_{+} ; 0,-\mu^{+}-\right.$ $\left.\mu^{\prime}\right),\left(\sigma_{M}^{\mu+\mu^{\prime}-l}(C)\right) \succcurlyeq P-T^{-\mu-\mu^{\prime}+l} Q$ for $l=0,1,2, \ldots$, and $G \in \Psi_{G ; P, Q}^{\mu^{\prime} ; 0}\left(\mathbb{R}_{+}\right)$. Furthermore, $A B\left(1-\zeta_{P}^{\mu^{\prime}}\right) \in \Psi_{G ; P, Q}^{\mu^{\prime} ; 0}\left(\mathbb{R}_{+}\right)$by Proposition 3.18. Since, obviously, $A B: \mathcal{S}_{P}\left(\mathbb{R}_{+}\right) \rightarrow \mathcal{S}_{Q}\left(\mathbb{R}_{+}\right)$, this all together shows that $A B \in \Psi_{P, Q}^{\mu+\mu^{\prime}, \mu^{\prime} ; 0}\left(\mathbb{R}_{+}\right)$.

\section{Further Elements of the Calculus}

In this section, the elements of the calculus are further developed. This concerns ellipticity and elliptic regularity, the parametrix construction for elliptic operators, and coordinate invariance.

4.1. Symbolic structure. The principal symbol space for $\Psi_{P, Q}^{\mu, d}\left(\mathbb{R}_{+} ; N_{-}, N_{+}\right)$ is introduced. 
Definition 4.1. For $\mu \in \mathbb{Z}, P, Q \in \underline{A s^{\sharp}}$, the space $\Sigma_{P, Q}^{\mu}$ consists of all couples $(m, a) \in \mathcal{M}_{\text {as }}^{\mu} \times S^{(\mu), 0}\left(\overline{\mathbb{R}}_{+} \times(\mathbb{R} \backslash 0)\right)$, where $(m(z)) \succcurlyeq P-T^{-\mu} Q$ and the compatibility condition

$$
\sigma_{\psi}^{\mu}(m)(-\tau)=a(0, \tau)
$$

(see $(2.2),($ A.5)) is fulfilled.

For $\mathcal{A} \in \Psi_{P, Q}^{\mu, d}\left(\mathbb{R}_{+} ; N_{-}, N_{+}\right)$represented in the form $(3.8)$, we define $\sigma_{\psi}^{\mu}(\mathcal{A})(t, \tau)$ to be the principal symbol of $A$ considered as operator in $L_{\mathrm{cl}}^{\mu}\left(\mathbb{R}_{+}\right)$and, further,

$$
\sigma_{M}^{\mu}(\mathcal{A})(z):=h(0, z)
$$

By construction, $\left(\sigma_{M}^{\mu}(\mathcal{A}), \sigma_{\psi}^{\mu}(\mathcal{A})\right) \in \Sigma_{P, Q}^{\mu}$ for $\mathcal{A} \in \Psi_{P, Q}^{\mu, d}\left(\mathbb{R}_{+} ; N_{-}, N_{+}\right)$.

Proof of Theorem 1.3. This proof follows exactly the lines of that one given for Proposition A.5 in Schulze [22, 23].

4.2. Ellipticity and parametrices. Ellipticity and the parametrix construction follow. As mentioned in the introduction, for a discussion of the ellitpicity assumtions, see Remarks 3.17, 4.3(a), and 4.11.

Definition 4.2. An operator $\mathcal{A} \in \Psi_{P, Q}^{\mu, d}\left(\mathbb{R}_{+} ; N_{-}, N_{+}\right)$represented in the form (3.8) is called elliptic if

(i) $\left(\sigma_{M}^{\mu}(\mathcal{A})(z)\right)=P-T^{-\mu} Q$;

(ii) $\sigma_{\psi}^{\mu}(\mathcal{A})(t, \tau) \neq 0$ for all $(t, \tau) \in \overline{\mathbb{R}}_{+} \times(\mathbb{R} \backslash 0)$;

(iii) $|p(t, \tau)| \geq C(1+|\tau|)^{\mu}$ for $|t|+|\tau| \geq R$ and some constants $C>0, R>0$.

Remark 4.3. (a) Due to the translation occurring in formula (A.4), it is necessary to require the lower bound in (3.1) on the whole of $\mathbb{C}$. Accordingly, also the requirement in Definition $4.2(\mathrm{i})$ also concerns the whole of $\mathbb{C}$.

(b) For $\mathcal{A} \in \Psi_{P, Q}^{\mu, d}\left(\mathbb{R}_{+} ; N_{-}, N_{+}\right)$, ellipticity only depends on the operator $A$ appearing in its upper-left corner. 
Next, we prepare for the parametrix construction.

Lemma 4.4. Let $\mathcal{A} \in \Psi_{P, Q}^{\mu, d}\left(\mathbb{R}_{+} ; N_{-}, N_{+}\right)$be elliptic. Then

$$
\left(\sigma_{M}^{\mu}(\mathcal{A})(z+\mu)^{-1}, \sigma_{\psi}^{\mu}(\mathcal{A})^{-1}\right) \in \Sigma_{Q, P}^{-\mu}
$$

with both inverses taken point-wise.

Lemma 4.5. If $\mathcal{A} \in \Psi_{P, Q}^{\mu, d}\left(\mathbb{R}_{+} ; N_{-}, N_{+}\right)$is elliptic in the sense of Definition 4.2 , then there is a $\mathcal{B} \in \Psi_{Q, P}^{-\mu, 0}\left(\mathbb{R}_{+} ; N_{+}, N_{-}\right)$such that

$$
\mathcal{B} \mathcal{A}-1 \in \Psi_{P}^{-\infty, \max \{\mu, d\}}\left(\mathbb{R}_{+} ; N_{-}, N_{-}\right), \quad \mathcal{A B}-1 \in \Psi_{Q}^{-\infty,(d-\mu)^{+}}\left(\mathbb{R}_{+} ; N_{+}, N_{+}\right) .
$$

Proof. By virtue of Theorem 1.3 and Lemma 4.4, we can choose a $\mathcal{B}_{0} \in$ $\Psi_{Q, P}^{-\mu, 0}\left(\mathbb{R}_{+} ; N_{+}, N_{-}\right)$such that

$$
\sigma_{M}^{-\mu}\left(\mathcal{B}_{0}\right)(z)=\sigma_{M}^{\mu}(\mathcal{A})(z+\mu)^{-1}, \quad \sigma_{\psi}^{-\mu}\left(\mathcal{B}_{0}\right)=\sigma_{\psi}^{\mu}(\mathcal{A})^{-1}
$$

Then, in view of Theorems 1.2, 1.3,

$$
\mathcal{N}_{0}:=1-\mathcal{B}_{0} \mathcal{A} \in \Psi_{P}^{-1, \max \{\mu, d\}}\left(\mathbb{R}_{+} ; N_{-}, N_{-}\right) .
$$

We get $\mathcal{N}_{0}{ }^{j} \in \Psi_{P}^{-j, \max \{\mu, d\}}\left(\mathbb{R}_{+} ; N_{-}, N_{-}\right)$for all $j \in \mathbb{N}, j \geq 1$, such that we can choose an $\mathcal{N} \in \Psi_{P}^{-1, \max \{\mu, d\}}\left(\mathbb{R}_{+} ; N_{-}, N_{-}\right)$satisfying

$$
\mathcal{N}-\sum_{j=1}^{J} \mathcal{N}_{0}^{j} \in \Psi_{P}^{-J-1, \max \{\mu, d\}}\left(\mathbb{R}_{+} ; N_{-}, N_{-}\right)
$$

for all $J \in \mathbb{N}$ according to Proposition 3.15. Then

$$
\mathcal{B}^{\prime}:=(1+\mathcal{N}) \mathcal{B}_{0} \in \Psi^{-\mu,(d-\mu)^{+}}\left(\mathbb{R}_{+} ; N_{+}, N_{-}\right)
$$

satisfies

$$
\mathcal{B}^{\prime} \mathcal{A}-1 \in \Psi_{P}^{-\infty, \max \{\mu, d\}}\left(\mathbb{R}_{+} ; N_{-}, N_{-}\right)
$$

Starting with $\mathcal{N}_{1}:=1-\mathcal{A B}_{0} \in \Psi_{Q}^{-1,(d-\mu)^{+}}\left(\mathbb{R}_{+} \times \Omega ; N_{+}, N_{+}\right)$in place of $\mathcal{N}_{0}$, in the same manner we get a $\mathcal{B}^{\prime \prime} \in \Psi_{Q, P}^{-\mu,(d-\mu)^{+}}\left(\mathbb{R}_{+} ; N_{+}, N_{-}\right)$satisfying

$$
\mathcal{A B}^{\prime \prime}-1 \in \Psi_{Q}^{-\infty,(d-\mu)^{+}}\left(\mathbb{R}_{+} ; N_{+}, N_{+}\right) .
$$


We finally have

$$
\mathcal{B}^{\prime}-\mathcal{B}^{\prime \prime}=\left(\mathcal{B}^{\prime} \mathcal{A}-1\right) \mathcal{B}^{\prime \prime}-\mathcal{B}^{\prime}\left(\mathcal{A B}^{\prime \prime}-1\right) \in \Psi_{Q, P}^{-\infty,(d-\mu)^{+}}\left(\mathbb{R}_{+} ; N_{+}, N_{-}\right),
$$

and upon writing $\mathcal{B}^{\prime}=\mathcal{B}+\mathcal{G}$, where $\mathcal{B} \in \Psi_{Q, P}^{-\mu, 0}\left(\mathbb{R}_{+} ; N_{+}, N_{-}\right)$and $\mathcal{G} \in$ $\Psi_{G ; Q, P}^{(d-\mu)^{+}}\left(\mathbb{R}_{+} ; N_{+}, N_{-}\right)$, we arrive at $\mathcal{B}$ as desired.

Now, we are able to conclude the proofs of Theorems 1.4, 1.5:

Proof of Theorem 1.4. Let $\mathcal{B}=\left(\begin{array}{c}B \\ T^{\prime} \\ K^{\prime}\end{array}\right) \in \Psi_{Q, P}^{-\mu, 0}\left(\mathbb{R}_{+} ; N_{+}, N_{-}\right)$be as constructed in the previous lemma. Let

$$
B=\omega t^{\mu} \operatorname{op}_{M}^{1 / 2-\delta}(k) \omega_{0}+(1-\omega) \operatorname{op}_{\psi}(\tilde{q})\left(1-\omega_{1}\right)+G^{\prime},
$$

where $k \in S_{\mathrm{cl}}^{-\mu}\left(\overline{\mathbb{R}}_{+} \times \Gamma_{1 / 2-\delta}\right)_{Q, P}, \tilde{q} \in S_{\mathrm{cl}}^{-\mu}\left(\mathbb{R}_{+} \times \mathbb{R}\right) \cap S^{-\mu ; 0}\left(\mathbb{R}_{+} \times \mathbb{R}\right)$, and $G^{\prime} \in \Psi_{G ; Q, P}^{0}\left(\mathbb{R}_{+} ; N_{+}, N_{-}\right)$, and we also assume that

$$
t^{\mu} \mathrm{op}_{M}^{1 / 2-\delta}(k)-\mathrm{op}_{\psi}(\tilde{q}) \in L^{-\infty}\left(\mathbb{R}_{+}\right) .
$$

By construction, $k \sharp_{M} h-1 \in S^{-\infty}\left(\overline{\mathbb{R}}_{+} \times \mathbb{R}\right)_{P, P}, \tilde{q} \sharp_{\psi} p-1 \in S^{-\infty ; 0}\left(\mathbb{R}_{+} \times \mathbb{R}\right)$, where $\mathcal{A}$ is given by (3.8). By requirement (iii) of Definition 4.2 , there is a $q \in S_{\mathrm{cl}}^{-\mu}\left(\mathbb{R}_{+} \times \mathbb{R}\right) \cap S^{-\mu ; 0}\left(\mathbb{R}_{+} \times \mathbb{R}\right)$ such that $q \sharp_{\psi} p-1 \in S^{-\infty ;-\infty}\left(\mathbb{R}_{+} \times \mathbb{R}\right)$. Because of $q-\tilde{q} \in S^{-\infty}\left(\mathbb{R}_{+} \times \mathbb{R}\right),(4.1)$ continues to hold with $q$ in place of $\tilde{q}$. Then $\mathcal{P}=\left(\begin{array}{cc}P & 0 \\ 0 & 0\end{array}\right) \in \Psi_{Q, P}^{-\mu, 0}\left(\mathbb{R}_{+} ; N_{+}, N_{-}\right)$with

$$
P=\omega t^{\mu} \mathrm{op}_{M}^{1 / 2-\delta}(k) \omega_{0}+(1-\omega) \mathrm{op}_{\psi}(q)\left(1-\omega_{1}\right)
$$

has the required properties.

As an immediate corollary, we obtain elliptic regularity:

Proposition 4.6. Let $\mathcal{A} \in \Psi_{P, Q}^{\mu, d}\left(\mathbb{R}_{+} ; N, N_{+}\right)$be elliptic and, for some s satisfying $s \geq \mu^{+}$, and $s>s_{d}$ when $d \geq d_{0}, u \in \mathbb{H}_{P}^{s, \delta}\left(\mathbb{R}_{+}\right), c \in \mathbb{C}^{N_{-}}$,

$$
\mathcal{A}\left(\begin{array}{l}
u \\
c
\end{array}\right) \in \mathbb{R}_{Q}^{\mathbb{H}_{Q}^{s^{\prime}-\mu, \delta}\left(\mathbb{R}_{+}\right)},
$$


where $s^{\prime}>s$. Then $u \in \mathbb{H}_{P}^{s^{\prime}, \delta}\left(\mathbb{R}_{+}\right)$.

Proof of Theorem 1.5. For some s, let $\mathcal{A}^{-1}$ be the inverse to $\mathcal{A}$ in (1.2). By elliptic regularity, $\mathcal{A}^{-1}$ is also the inverse to $\mathcal{A}$ considered as operator in (3.9). Let $\mathcal{P} \in \Psi_{Q, P}^{-\mu, 0}\left(\mathbb{R}_{+} ; N_{+}, N_{-}\right)$be a parametrix to $\mathcal{A}$ and $\mathcal{G}:=\mathcal{A} \mathcal{P}-1 \in$ $\Psi_{G ; Q}^{(d-\mu)^{+}}\left(\mathbb{R}_{+} ; N_{+}, N_{+}\right)$. Due to the explicit description given in Definition 3.10, it is seen that $\mathcal{A}^{-1} \mathcal{G} \in \Psi_{G ; Q, P}^{(d-\mu)^{+}}\left(\mathbb{R}_{+} ; N_{+}, N_{-}\right)$. Then

$$
\mathcal{A}^{-1}=\mathcal{P}-\mathcal{A}^{-1} \mathcal{G}
$$

showing that $\mathcal{A}^{-1} \in \Psi_{Q, P}^{-\mu,(d-\mu)^{+}}\left(\mathbb{R}_{+} ; N_{+}, N_{-}\right)$.

4.3. Coordinate invariance. Finally, we come to the discussion of coordinate invariance of the calculus. Let $\chi: \overline{\mathbb{R}}_{+} \rightarrow \overline{\mathbb{R}}_{+}$be a $C^{\infty}$-diffeomorphism such that $\left|\chi^{(j)}(t)\right| \leq C_{j}(1+t)^{1-j}$ for all $t \geq 0, j \in \mathbb{N}$, and certain constants $C_{j}>0$ and $\chi(t) \geq c t$ for all $t \geq 0$ and some $c>0$.

First of all, we have:

Lemma 4.7. Let $P \in \underline{A s}^{\delta}$ satisfy the shadow condition. Then $\chi_{*}: \mathbb{H}_{P}^{s, \delta}\left(\mathbb{R}_{+}\right) \rightarrow$ $\mathbb{H}_{P}^{s, \delta}\left(\mathbb{R}_{+}\right)$.

From KaPANADZE-SchulzE-WitT [10], we quote the following result concerning the transformation rules for complete Mellin symbols and thus accompanying Proposition A.8:

For $j \in \mathbb{N}$, we define

$$
\Delta_{j} h(z):=z(h(z)-h(z-j)), \quad h \in \mathcal{M}_{\mathrm{as}}^{\mu} .
$$

Note that $\Delta_{j}: \mathcal{M}_{\text {as }}^{\mu} \rightarrow \mathcal{M}_{\text {as }}^{\mu}$, since $h(z), h(z-j)$ for $h \in \mathcal{M}_{\text {as }}^{\mu}$ possess the same principal symbol. For $j_{1}, j_{2}, \ldots, j_{m} \in \mathbb{N}, j_{1}, j_{2}, \ldots, j_{m} \geq 1$, we further define

$$
\begin{aligned}
& \Delta\left(\mu ; j_{1}, j_{2}, \ldots, j_{m}\right):=\left(\Delta_{j_{1}}+\mu-\left(j_{2}+j_{3}+\cdots+j_{m}\right)\right) \times \\
&\left(\Delta_{j_{2}}+\mu-\left(j_{3}+\cdots+j_{m}\right)\right) \ldots\left(\Delta_{j_{m-1}}+\mu-j_{m}\right)\left(\Delta_{j_{m}}+\mu\right) .
\end{aligned}
$$


Lemma 4.8. For $j, k \in \mathbb{N}, j, k \geq 1$,

$$
\left(\Delta_{j}+\mu-k\right)\left(\Delta_{k}+\mu\right)-\left(\Delta_{k}+\mu-j\right)\left(\Delta_{j}+\mu\right)=(j-k)\left(\Delta_{j+k}+\mu\right)
$$

Therefore, the parameters in defining the operators $\Delta\left(\mu ; j_{1}, j_{2}, \ldots, j_{m}\right)$ can be commuted freely; up to linear combinations of operators of the same kind. Especially, we may assume that $j_{1} \leq j_{2} \leq \cdots \leq j_{m}$. We then introduce:

$$
\Delta\left[\mu ; a_{1}, a_{2}, \ldots, a_{j}\right]:=\Delta(\mu ; \underbrace{1, \ldots, 1}_{a_{1} \text { times }}, \underbrace{2, \ldots, 2}_{a_{2} \text { times }}, \ldots, \underbrace{j, \ldots, j}_{a_{j} \text { times }}) .
$$

Proposition 4.9. There exist universal polynomials $q_{j}\left(y_{1}, \ldots, y_{j}\right)$ for $j=$ $1,2, \ldots$ such that, for each $A \in C^{\mu}\left(\mathbb{R}_{+} ; \gamma, \gamma-\mu\right)$,

$$
\begin{aligned}
& \text { (4.2) } \sigma_{M}^{\mu-j}\left(A_{\chi}\right)(z)=\chi^{\prime}(0)^{\mu-j}\left\{\sigma_{M}^{\mu-j}(A)(z)+\sum_{r=0}^{j-1}\right. \\
& \left.\times \sum_{a_{1}+2 a_{2}+\ldots+(j-r) a_{j-r}=j-r} \frac{\bar{q}_{1}^{a_{1}} \bar{q}_{2}^{a_{2}} \ldots \bar{q}_{j-r}^{a_{j-r}}}{a_{1} ! a_{2} ! \ldots a_{j-r} !} \Delta\left[\mu-r ; a_{1}, a_{2}, \ldots, a_{j-r}\right] \sigma_{M}^{\mu-r}(A)(z)\right\}
\end{aligned}
$$

for $j=0,1,2, \ldots$, where $A_{\chi}$ is the operator push-forward of $A$ under $\chi$. Here

$$
\bar{q}_{j}(\chi)=q_{j}\left(\frac{\chi^{\prime \prime}(0)}{\chi^{\prime}(0)}, \frac{\chi^{(3)}(0)}{\chi^{\prime}(0)}, \ldots, \frac{\chi^{(j+1)}(0)}{\chi^{\prime}(0)}\right) .
$$

Remark 4.10. The first of the polynomials $q_{j}\left(y_{1}, y_{2}, \ldots, y_{j}\right)$ are

$$
\begin{aligned}
& q_{1}=\frac{1}{2} y_{1}, \quad q_{2}=-\frac{1}{4} y_{1}^{2}+\frac{1}{6} y_{2}, \quad q_{3}=\frac{1}{8} y_{1}^{3}-\frac{1}{6} y_{1} y_{2}+\frac{1}{24} y_{3}, \\
& q_{4}=-\frac{3}{32} y_{1}^{4}-\frac{1}{24} y_{1} y_{3}+\frac{1}{6} y_{1}^{2} y_{2}-\frac{1}{24} y_{2}^{2}+\frac{1}{120} y_{4}, \\
& q_{5}=\frac{1}{16} y_{1}^{5}-\frac{7}{48} y_{1}^{3} y_{2}+\frac{5}{72} y_{1} y_{2}^{2}+\frac{1}{24} y_{1}^{2} y_{3}-\frac{1}{48} y_{2} y_{3}-\frac{1}{120} y_{1} y_{4}+\frac{1}{720} y_{5} .
\end{aligned}
$$

Proof of Theorem 1.6. By virtue of Remark 3.4, Lemma 3.19 and Propositions $3.20,4.9, \mathrm{~A} .8$, it suffices to prove that, for $\mu \geq \nu$ and $h(z) \in \mathcal{M}_{\mathcal{O}}^{\mu-\nu}$,

$$
\Delta\left(\mu ; j_{1}, \ldots, j_{m}\right)\left(h(z) \frac{g_{Q}(z+\mu)}{g_{P}(z)}\right)=k(z) \frac{g_{Q}(z+\mu)}{g_{P}(z)}
$$

for some $k(z) \in \mathcal{M}_{\mathcal{O}}^{\mu-\nu}$, where $j_{1}+\cdots+j_{m}=\mu-\nu$. 
We proceed by induction on $\mu-\nu$. For $\mu-\nu=0$, there is nothing to do. Next we consider, for $j_{0}+j_{1}+\cdots+j_{m}=\mu-\nu$ and $h(z) \in \mathcal{M}_{\mathcal{O}}^{\mu-\nu}$,

$$
\begin{aligned}
\Delta\left(\mu ; j_{0}, j_{1}, \ldots, j_{m}\right)\left(h(z) \frac{g_{Q}(z+\mu)}{g_{P}(z)}\right) \\
\quad=\left(\Delta_{j_{0}}+\left(\mu-\left(j_{1}+\cdots+j_{m}\right)\right) \Delta\left(\mu ; j_{1}, \ldots, j_{m}\right)\left(h(z) \frac{g_{Q}(z+\mu)}{g_{P}(z)}\right)\right. \\
=\left(\Delta_{j_{0}}+\nu+j_{0}\right)\left(\tilde{h}(z) \frac{g_{Q}\left(z+\nu+j_{0}\right)}{g_{P}(z)}\right) \\
=\left(z+\nu+j_{0}\right) \tilde{h}(z) \frac{g_{Q}\left(z+\nu+j_{0}\right)}{g_{P}(z)}-z \tilde{h}\left(z-j_{0}\right) \frac{g_{Q}(z+\nu)}{g_{P}\left(z-j_{0}\right)} \\
=k(z) \frac{g_{Q}(z+\mu)}{g_{P}(z)},
\end{aligned}
$$

where $\tilde{h}(z) \in \mathcal{M}_{\mathcal{O}}^{\mu-\nu-j_{0}}$ by inductive hypotheses and

$$
k(z):=\left(z+\nu+j_{0}\right) \tilde{h}(z) \frac{g_{Q}\left(z+\nu+j_{0}\right)}{g_{Q}(z+\nu)}-z \tilde{h}\left(z-j_{0}\right) \frac{g_{P}(z)}{g_{P}\left(z-j_{0}\right)} .
$$

Here, both summands on the right-hand side belong to $\mathcal{M}_{\mathcal{O}}^{\mu-\nu+1}$ (in particular, they are holomorphic, since $P, Q$ satisfy the shadow condition), but their difference belongs to $\mathcal{M}_{\mathcal{O}}^{\mu-\nu}$, since both summands have the same principal symbol (by the choice made in Proposition 2.7).

This finishes the proof.

Remark 4.11. To require

$$
\sigma_{M}^{\mu}(\mathcal{A})(z)=a_{0} \frac{g_{Q}(z+\mu)}{g_{P}(z)}, \quad a_{0} \in \mathbb{C},
$$

instead of (3.1) for $j=0$, see (3.2), also leads to a coordinate invariant calculus. There is, however, no canonical choice for the functions $g_{P}(z), g_{Q}(z)$; unlike the case $P=Q=P_{0}$, in which we can choose $g_{P_{0}}(z)=\Gamma(z)$.

\section{Appendix A. Schulze's cone calculus}

We briefly introduce Schulze's cone calculus on $\mathbb{R}_{+}$. For further details, we refer to ScHulzE [22, 23]. 
For $s \in \mathbb{N}, \gamma \in \mathbb{R}, \mathcal{H}^{s, \gamma}\left(\mathbb{R}_{+}\right)$is defined to be the space of all $u \in L_{\text {loc }}^{2}\left(\mathbb{R}_{+}\right)$such that

$$
t^{j-\gamma} \partial_{t}^{j} u \in L^{2}\left(\mathbb{R}_{+}\right), \quad j \leq s
$$

For general $s, \gamma \in \mathbb{R}$, the spaces $\mathcal{H}^{s, \gamma}\left(\mathbb{R}_{+}\right)$are then defined by interpolation and duality. In terms of the Mellin transformation, $\mathcal{H}^{s, \gamma}\left(\mathbb{R}_{+}\right)$consists of all $u$ for which $\tilde{u} \in L_{\mathrm{loc}}^{2}\left(\mathbb{R}_{+}\right)$and

$$
\|u\|_{\mathcal{H}^{s, \gamma}\left(\mathbb{R}_{+}\right)}:=\left\{\frac{1}{2 \pi i} \int_{z \in \Gamma_{1 / 2-\delta}}\langle z\rangle^{2 s}|\tilde{u}(z)|^{2} d z\right\}^{1 / 2}<\infty .
$$

Furthermore, we define

$$
\mathcal{K}^{s, \gamma}\left(\mathbb{R}_{+}\right):=\left\{u \in H_{\mathrm{loc}}^{s}\left(\mathbb{R}_{+}\right) ; \omega u \in \mathcal{H}^{s, \gamma}\left(\mathbb{R}_{+}\right),(1-\omega) \in H^{s}\left(\mathbb{R}_{+}\right)\right\}
$$

where $\omega=\omega(t)$ is some cut-off function. $\mathcal{K}^{s, \gamma}\left(\mathbb{R}_{+}\right)$is a Hilbert space with its norm given by

$$
\|u\|_{\mathcal{K}^{s, \gamma}\left(\mathbb{R}_{+}\right)}:=\left\{\|\omega u\|_{\mathcal{H}^{s, \gamma}\left(\mathbb{R}_{+}\right)}^{2}+\|(1-\omega) u\|_{H^{s\left(\mathbb{R}_{+}\right)}}^{2}\right\}^{1 / 2} .
$$

We have $\mathcal{K}^{0,0}\left(\mathbb{R}_{+}\right)=L^{2}\left(\mathbb{R}_{+}\right), \mathcal{K}^{s, \gamma}\left(\mathbb{R}_{+}\right) \subseteq \mathcal{K}^{s^{\prime}, \gamma^{\prime}}\left(\mathbb{R}_{+}\right)$if and only if $s \geq s^{\prime}$, $\gamma \geq \gamma^{\prime}$, and $\mathcal{K}^{-s,-\gamma}\left(\mathbb{R}_{+}\right)$is the dual space to $\mathcal{K}^{s, \gamma}\left(\mathbb{R}_{+}\right)$with respect to the $L^{2}$-scalar product.

For $P \in \underline{A s^{\gamma}}, \vartheta>0$, we define

$$
\begin{aligned}
& \mathcal{K}_{P, \vartheta-0}^{s, \gamma}\left(\mathbb{R}_{+}\right):=\left\{\sum_{\substack{k+1 \leq P(p), \operatorname{Re} p>1 / 2-\gamma-\vartheta}} \omega(t) t^{-p} \log ^{k} t c_{p k} ; c_{p k} \in \mathbb{C}\right\} \oplus \bigcap_{\epsilon>0} \mathcal{K}^{s, \gamma+\vartheta-\epsilon}\left(\mathbb{R}_{+}\right) \\
& \mathcal{S}_{P, \vartheta-0}^{\gamma}\left(\mathbb{R}_{+}\right):=\left\{u \in C^{\infty}\left(\mathbb{R}_{+}\right) ; \omega u \in \bigcap_{s \in \mathbb{R}} \mathcal{K}_{P, \vartheta-0}^{s, \gamma}\left(\mathbb{R}_{+}\right),(1-\omega) u \in \mathcal{S}\left(\overline{\mathbb{R}}_{+}\right)\right\} .
\end{aligned}
$$

Note that $\mathcal{S}_{P, \infty}^{\gamma}\left(\mathbb{R}_{+}\right):=\bigcap_{\vartheta>0} \mathcal{S}_{P, \vartheta-0}^{\gamma}\left(\mathbb{R}_{+}\right)$equals the space $\mathcal{S}_{P}\left(\mathbb{R}_{+}\right)$defined in $(2.9)$.

Definition A.1. Let $\mu, \nu \in \mathbb{Z}, \mu \geq \nu, \gamma, \delta \in \mathbb{R}, k \in \mathbb{N} \cup\{\infty\}$, and $\vartheta_{k}:=k+1$.

(i) The space $C_{G}\left(\mathbb{R}_{+} ; \gamma, \delta, \vartheta_{k}-0\right)$ consists of all operators $G: \mathcal{K}^{s, \gamma}\left(\mathbb{R}_{+}\right) \rightarrow$ $\mathcal{S}_{P, \vartheta_{k}-0}^{\delta}\left(\mathbb{R}_{+}\right)$such that $G^{*}: \mathcal{K}^{s,-\delta}\left(\mathbb{R}_{+}\right) \rightarrow \mathcal{S}_{Q, \vartheta_{k}-0}^{-\gamma}\left(\mathbb{R}_{+}\right)$for certain $P \in \underline{\text { As }^{\delta}}$, 
$Q \in \underline{\mathrm{As}}^{-\gamma}$, where $G^{*}$ is the formally adjoint to $G$ with respect to the $L^{2}$-scalar product.

(ii) For $k \in \mathbb{N}$, the space $C^{\nu}\left(\mathbb{R}_{+} ; \gamma, \gamma-\mu, \vartheta_{k}-0\right)$ consists of all operators $A$ on $\mathbb{R}_{+}$of the form

$$
\begin{array}{r}
A=\omega(t) t^{-\nu} \operatorname{op}_{M}^{1 / 2-\delta}(h(t, z)) \omega_{0}(t)+\omega(t) t^{-\nu} \sum_{j=0}^{k-\mu+\nu} t^{j} \operatorname{op}_{M}^{1 / 2-\gamma+\rho_{j}}\left(h_{j}(z)\right) \omega(t) \\
+(1-\omega(t)) \operatorname{op}_{\psi}(p)\left(1-\omega_{1}(t)\right)+G,
\end{array}
$$

where $h \in C^{\infty}\left(\overline{\mathbb{R}}_{+} ; \mathcal{M}_{\mathcal{O}}^{\nu}\right), h_{j} \in \mathcal{M}_{R_{j}}^{-\infty}$ for certain $R_{j} \in \underline{\text { As }}, 0 \leq \rho_{j} \leq j$, $\Gamma_{1 / 2-\gamma+\rho_{j}} \cap \pi_{\mathbb{C}} R_{j}=\emptyset$ for $j=0,1, \ldots, k-\mu+\nu, p \in S_{\text {cl }}^{\nu}\left(\mathbb{R}_{+} \times \mathbb{R}\right) \cap S^{\nu ; 0}\left(\mathbb{R}_{+} \times \mathbb{R}\right)$, and $G \in C_{G}\left(\mathbb{R}_{+} ; \gamma, \gamma-\mu, \vartheta_{k}-0\right) ; \omega, \omega_{0}, \omega_{1}$ satisfying $\omega \omega_{0}=\omega, \omega \omega_{1}=\omega_{1}$ are cut-off functions as before.

(iii) We finally set

$$
C^{\nu}\left(\mathbb{R}_{+} ; \gamma, \gamma-\mu\right):=\bigcap_{k \in \mathbb{N}} C^{\nu}\left(\mathbb{R}_{+} ; \gamma, \gamma-\mu, \vartheta_{k}-0\right)
$$

Note that $C^{\nu}\left(\mathbb{R}_{+} ; \gamma, \gamma-\mu\right) \subset L_{\text {cl }}^{\nu}\left(\mathbb{R}_{+}\right) \cap L^{\nu ; 0}\left(\mathbb{R}_{+}\right)$. In case $k=\infty$, we also write $C_{G}\left(\mathbb{R}_{+} ; \gamma, \delta\right)$ instead of $C_{G}\left(\mathbb{R}_{+} ; \gamma, \delta, \vartheta_{\infty}-0\right)$.

Proposition A.2. For all $A \in C^{\mu}\left(\mathbb{R}_{+} ; \gamma, \gamma-\mu\right), P \in \underline{\text { As }^{\gamma}}$, there is a $Q \in$ $\underline{\mathrm{As}}^{\gamma-\mu}$ such that $A$ induces continuous operators

$$
A: \mathcal{K}^{s, \gamma}\left(\mathbb{R}_{+}\right) \rightarrow \mathcal{K}^{s-\mu, \gamma-\mu}\left(\mathbb{R}_{+}\right)
$$

and

$$
A: \mathcal{K}_{P, \vartheta-0}^{s, \gamma}\left(\mathbb{R}_{+}\right) \rightarrow \mathcal{K}_{Q, \vartheta-0}^{s-\mu, \gamma-\mu}\left(\mathbb{R}_{+}\right)
$$

for all $s \in \mathbb{R}, \vartheta>0$. 
With an operator $A \in C^{\mu}\left(\mathbb{R}_{+} ; \gamma, \gamma-\mu\right)$, we associate its pseudodifferential (or interior) principal symbol

$$
\sigma_{\psi}^{\mu}(A)(t, \tau):=\omega(t) \sigma_{\psi}^{\mu}(h(t, \cdot))(-\tau)+(1-\omega(t)) \sigma_{\psi}^{\mu}\left(A_{\psi}\right)(t, \tau)
$$

and its complete Mellin symbol $\left\{\sigma_{M}^{\mu-j}(A)(z) ; j \in \mathbb{N}\right\}$, where

$$
\sigma_{M}^{\mu-j}(A)(z):=\frac{1}{j !} \frac{\partial^{j} h}{\partial t^{j}}(0, z)+h_{j}(z)
$$

for $j=0,1,2, \ldots$, and $A$ is given in the form (A.1) with $k$ sufficiently large (and $\nu=\mu$ ). These symbol are uniquely determined, i.e., they are independent of the chosen representation (A.1).

Proposition A.3. Let $A \in C^{\nu}\left(\mathbb{R}_{+} ; \gamma-\mu^{\prime}, \gamma-\mu-\mu^{\prime}\right), B \in C^{\nu^{\prime}}\left(\mathbb{R}_{+} ; \gamma, \gamma-\mu^{\prime}\right)$ for some $\mu \geq \nu, \mu^{\prime} \geq \nu^{\prime}$. Then $A B \in C^{\nu+\nu^{\prime}}\left(\mathbb{R}_{+} ; \gamma, \gamma-\mu-\mu^{\prime}\right)$. Furthermore, in case $\mu=\nu, \mu^{\prime}=\nu^{\prime}, \sigma_{\psi}^{\mu+\mu^{\prime}}(A B)=\sigma_{\psi}^{\mu}(A) \sigma_{\psi}^{\mu^{\prime}}(B)$ as well as

$$
\sigma_{M}^{\mu+\mu^{\prime}-l}(A B)(z)=\sum_{j+k=l} \sigma_{M}^{\mu-j}(A)\left(z+\mu^{\prime}-k\right) \sigma_{M}^{\mu^{\prime}-k}(B)(z)
$$

for $l=0,1,2, \ldots$

(A.4) is called the Mellin translation product.

Definition A.4. The symbol space $\Sigma^{\mu}$ consists of all couples $(m, a) \in \mathcal{M}_{\text {as }}^{\mu} \times$ $S^{(\mu) ; 0}\left(\overline{\mathbb{R}}_{+} \times(\mathbb{R} \backslash 0)\right)$ such that the compatibility condition

$$
\sigma_{\psi}^{\mu}(m)(-\tau)=a(0, \tau)
$$

(see $(2.2))$ is fulfilled.

Proposition A.5. The short sequence

$$
0 \longrightarrow C^{\mu-1}\left(\mathbb{R}_{+} ; \gamma, \gamma-\mu\right) \longrightarrow C^{\mu}\left(\mathbb{R}_{+} ; \gamma, \gamma-\mu\right) \stackrel{\left(\sigma_{M}^{\mu}, \sigma_{\psi}^{\mu}\right)}{\longrightarrow} \Sigma^{\mu} \longrightarrow 0
$$

is split exact. 
Definition A.6. An operator $A \in C^{\mu}\left(\mathbb{R}_{+} ; \gamma, \gamma-\mu\right)$ is called elliptic if

(i) $\sigma_{M}^{\mu}(A)(z) \neq 0$ for all $z \in \Gamma_{1 / 2-\gamma}$;

(ii) $\sigma_{\psi}^{\mu}(A)(t, \tau) \neq 0$ for all $(t, \tau) \in \overline{\mathbb{R}}_{+} \times(\mathbb{R} \backslash 0)$;

(iii) There are constants $C>0, R>0$ such that $|p(t, \tau)| \geq C(1+|\tau|)^{\mu}$ for all $(t, \tau) \in \mathbb{R}_{+} \times \mathbb{R},|t|+|\tau| \geq R$, where $A$ is given the form (A.1).

Proposition A.7. An operator $A \in C^{\mu}\left(\mathbb{R}_{+} ; \gamma, \gamma-\mu\right)$ is elliptic if and only if $A$ regarded as operator in (A.3) is Fredholm for some $s \in \mathbb{R}$ (and then for all $s \in \mathbb{R})$. In such a case, a parametrix $P$ to $A$ exists, i.e., an operator $P \in C^{-\mu}\left(\mathbb{R}_{+} ; \gamma-\mu, \gamma\right)$ such that

$$
P A-1 \in C_{G}\left(\mathbb{R}_{+} ; \gamma, \gamma\right), \quad A P-1 \in C_{G}\left(\mathbb{R}_{+} ; \gamma-\mu, \gamma-\mu\right) .
$$

From KAPANADZE-SCHULZE-WiTT [10], we quote the following result:

Proposition A.8. The cone calculus $C^{\nu}\left(\mathbb{R}_{+} ; \gamma, \gamma-\mu\right)$ for $\mu \geq \nu$ is invariant under the operator push-forward under diffeomorphism $\chi$ as considered in Theorem 1.6 .

\section{ACKNOWLEDGEMENT}

This paper is in part an extended version of the first author's thesis. The first author is grateful to Professor Chen Hua from Wuhan University and Professor B.-W. Schulze from University of Potsdam for supervising her thesis and all the encouraging support during that time. Both authors thank Thomas Krainer and Jörg Seiler from University of Potsdam for a lot of fruitful discussion.

\section{REFERENCES}

1. L. Boutet de Monvel, Boundary problems for pseudo-differential operators, Acta Math. 126 (1971), 11-51.

2. C. Dorschfeldt, Algebras of pseudo-differential operators near edge and corner singularities, Math. Res., vol. 102, Akademie Verlag, Berlin, 1998. 
3. G. I. Eskin, Boundary value problems for elliptic pseudodifferential equations, Transl. Math. Monogr., vol. 52, Amer. Math. Soc, Providence, RI, 1980.

4. I. Gohberg and N. Krupnik, One-dimensional linear singular integral equations, Vol. I, Oper. Theory Adv. Appl., vol. 53, Birkhäuser, Basel, 1992.

5. I. C. Gohberg and E. I. Sigal, An operator generalization of the logarithmic residue theorem and the theorem of Rouché, Math. USSR Sbornik 13 (1971), 603-625.

6. G. Grubb, Functional calculus of pseudo-differential boundary problems, second ed., Progr. Math., vol. 65, Birkhäuser Boston, Boston, MA, 1996.

7. T. Hirschmann, Pseudo-differential operators and asymptotics on manifolds with corners. Supplement: Pseudo-differential operator calculus with exit conditions, Tech. Report R-MATH-04/91, Karl-Weierstrass-Institut für Mathematik, Berlin, 1991.

8. L. Hörmander, The analysis of linear differential operators III-IV, Grundlehren Math. Wiss., vols. 274-275, Springer, Berlin, 1985.

9. P. Jeanquartier, Transformation de Mellin et développements asymptotiques, Enseign. Math. 25 (1979), 285-308.

10. D. Kapanadze, B.-W. Schulze, and I. Witt, Coordinate invariance of the cone algebra with asymptotics, to appear in Advances in Partial Differential Equations.

11. V. A. Kondrat'ev, Boundary problems for elliptic equations in domains with conical or angular points, Trans. Mosc. Math. Soc. 16 (1967), 227-313.

12. H. Kumano-go, Pseudo-differential operators, The MIT Press, Cambridge, Massachusetts, 1981.

13. X. Liu, A cone pseudodifferential calculus on the half-line with respect to conormal asymptotics of a given type, Ph.D. thesis, Department of Mathematics, Wuhan University, Wuhan, 2000.

14. X. Liu and I. Witt, Asymptotic expansions for bounded solutions to semilinear Fuchsian equations, submitted.

15. R. B. Melrose, Transformation of boundary value problems, Acta Math. 147 (1981), $149-236$

16. The Atiyah-Patodi-Singer index theorem, Res. Notes Math., A K Peters, Wellesley MA, Boston, 1993.

17. F. W. J. Olver, Introduction to asymptotics and special functions, Academic Press, New York, 1974.

18. S. Rempel and B.-W. Schulze, Index theory of elliptic boundary problems, Math. Lehrbücher Monogr. II Abt. Math. Monogr., vol. 55, Akademie-Verlag, Berlin, 1982. 
19. Asymptotics for elliptic mixed boundary problems, Math. Res., vol. 50, Akademie-Verlag, Berlin, 1989.

20. E. Schrohe, Spaces of weighted symbols and weighted Sobolev spaces on manifolds, Pseudo-Differential Operators (Berlin) (H. O. Cordes, B. Gramsch, and H. Widom, eds.), Lecture Notes in Math., vol. 1256, Springer, 1987, pp. 360-377.

21. B.-W. Schulze, Pseudo-differential operators on manifolds with singularities, Stud. Math. Appl., vol. 24, North-Holland, Amsterdam, 1991.

22. Pseudo-differential boundary value problems, conical singularities, and asymptotics, Math. Top., vol. 4, Akademie Verlag, Berlin, 1994.

23 . Boundary value problems and singular pseudo-differential operators, Wiley Ser. Pure Appl. Math., J. Wiley, Chichester, 1998.

24. B.-W. Schulze and J. Seiler, The edge algebra structure of boundary value problems, Preprint 2001/11, Institute of Mathematics, University of Potsdam, Potsdam, 2001.

25. F. Treves, Topological vector spaces, distributions and kernels, Pure Appl. Math., vol. 25, Academic Press, San Diego, 1967.

26. I. Witt, Explicit algebras with the Leibniz-Mellin translation product, submitted.

27. On the factorization of meromorphic Mellin symbols, to appear in Advances in Partial Differential Equations.

28. , The weak symbol topology and continuity of pseudodifferential operators, Differential Equations, Asymptotic Analysis, and Mathematical Physics (Berlin) (M. Demuth and B.-W. Schulze, eds.), Math. Res., vol. 100, Akademie Verlag, 1997, pp. 412-422. Asymptotic algebras, Microlocal Analysis and Asymptotic Analysis of PDE (Kyoto), RIMS Kokyuroku, vol. 1211, Research Institute for Mathematical Sciences, Kyoto University, 2001, pp. 21-33. 